\title{
Magnetic reversal of a quantum nanoferromagnet
}

\author{
J. P. Gauyacq ${ }^{1}$ and N. Lorente ${ }^{2}$ \\ ${ }^{1}$ Institut des Sciences Moléculaires d'Orsay, ISMO, Unité Mixte de Recherches CNRS-Université Paris-Sud, UMR 8214, \\ Bâtiment 351, Université Paris-Sud, 91405 Orsay Cedex, France \\ ${ }^{2}$ Centre d'Investigació en Nanociéncia i Nanotecnologia (CSIC-ICN), Campus de la UAB, E-08193 Bellaterra, Spain
}

(Received 8 February 2013; published 1 May 2013)

\begin{abstract}
When the external magnetic field applied to a ferromagnetically coupled atomic chain is reversed suddenly, the magnetization of the chain switches, due to the reversal of all the atomic magnetic moments in the chain. The quantum processes underlying the magnetization switching and the time required for the switching are analyzed for model magnetic chains adsorbed on a surface at $0 \mathrm{~K}$. The sudden field reversal brings the chain into an excited state that relaxes towards the system ground state via interactions with the substrate electrons. Different mechanisms are outlined, ranging from the global stepwise rotation of the chain macrospin induced by spin-flip collisions with substrate electrons in the pure Heisenberg chain (Néel-Brown process) to a correlation-mediated direct switching process in the presence of strong magnetic anisotropies in short chains (the global spin of the chain reverses in a single electron interaction). The processes for magnetization switching induced by electrons tunneling from a scanning tunneling microscope tip are also analyzed.
\end{abstract}

DOI: 10.1103/PhysRevB.87.195402

PACS number(s): 68.37.Ef, 75.60.Jk, 75.75.Jn, 72.25.-b

\section{INTRODUCTION}

The fast development of miniaturization of electronic devices prompted an active series of studies on the structure and dynamics of small low-dimensional magnetic objects adsorbed on surfaces. Among these, the magnetization reversal of small objects received much attention because of its links with data storage, and indeed, it is quite important to understand and be able to represent the processes underlying the reversal. The magnetization reversal in ferromagnetic materials (magnetic switch of small magnetic domains) was described theoretically very early by Neel and Brown. ${ }^{1,2}$ Basically, the idea is that all the magnetic moments are aligned in small magnetic domains to form a classical macrospin that rotates as a whole. The dynamics of the spin can be described within the Landau-Lifshitz-Gilbert equation and lead to a description of the thermally induced magnetization reversal process. The development of spin-polarized scanning tunneling microscopy (SP-STM) led to detailed observations of the thermally induced magnetic switch in small ferromagnets, allowing a detailed test of the global rotation of the Néel-Brown view and of its limits. ${ }^{3-10}$ In parallel, analyses led to the discussion of other views of the thermally activated magnetization switch, ${ }^{10-17}$ for example, spin-wave contributions, nucleation, edge effects, and anisotropy effect. Following earlier works on multilayered materials, ${ }^{18,19}$ SP-STM experiments revealed that injection of spin-polarized electrons into a nanoferromagnet could also switch its magnetization. ${ }^{20-24}$ A similar process has been observed and modeled recently on adsorbed antiferromagnetic chains: The switch between the two Néel states of the chain can be induced by tunneling electrons from an STM tip. ${ }^{25,26}$ These works open the way toward the fascinating possibility of controlling the magnetization of small objects at surfaces. Various processes have been proposed and discussed for the electron-induced magnetic reversal: ${ }^{20-24}$ spin-transfer torque (the incident electron brings spin momentum to the object), Joule heating (the injected electrons locally heat the object, speeding up thermal reversal), and the Oerstedt field effect (the injected electron current generates a magnetic field that can influence the reversal), all possibly associated with quantum tunneling.

Recently, series of experimental ${ }^{27-30}$ and theoretical ${ }^{31-37}$ works have been reported on magnetic transitions induced by tunneling electrons in individual adsorbates, leading toward a full understanding of the magnetic dynamics at the atomic level and their possible control by electrons. A similar process has also been studied experimentally: the relaxation of excited magnetic states in adsorbates. ${ }^{38-40}$ Indeed, in the case of supported magnetic objects, electrons from the substrate continuously collide with adsorbates and go back into the substrate, and they can induce magnetic transitions in the adsorbate in a similar manner as tunneling electrons; in this way, an excited magnetic state can be de-excited by an electron-hole pair creation ${ }^{35,41,42}$ (see a review in Ref. 43). In both cases (excitation and de-excitation), an electron, possibly polarized, colliding on a magnetic adsorbate induces magnetic transitions, i.e., changes the magnetic state of the adsorbate. This aspect bears strong resemblances with the problem of magnetization switch in small objects briefly discussed above; it is either induced by thermal processes (interaction with a thermal bath, the substrate electrons) or by tunneling electrons. The theoretical works devoted to the analysis of this excitation/de-excitation process in individual adsorbates at a surface have been based on full quantum microscopic approaches of the nanomagnetic properties. The aim of the present work is to see how these quantum microscopic studies can be transferred to studies of magnetization reversal in adsorbed nanoferromagnets and how they can describe the corresponding processes.

In the present work, we report on a theoretical model study of the magnetization switch of a small ferromagnetic object, an adsorbed chain of atoms, under the reversal of the direction of an applied magnetic field, B. The small object is initially at equilibrium at $0 \mathrm{~K}$ for a finite $\mathrm{B}$ field pointing in a given direction, and the B field direction is suddenly reversed. Such an inversion of an applied field, similar to a quantum quench, allows us to study the fast time behavior far from equilibrium of 
a small ferromagnetic domain. The domain switch, both via the interaction with substrate electrons and via electrons injected by an STM tip, is discussed. Our study considers finite chains of atomic spins and is of model character. It is based on the strong coupling approach recently introduced to treat magnetic transitions induced by tunneling electrons in adsorbates and relaxation of magnetic excitation by electron-hole pair creation in the substrate. ${ }^{34,41,43,44}$

\section{METHOD}

The present work uses the strong coupling approach, which has been developed to treat the excitation of individual magnetic adsorbates at surfaces by tunneling electrons ${ }^{34,43,44}$ and extended to treat the electron-induced magnetic excitations in chains ${ }^{45}$ as well as the lifetime of magnetic excitations. ${ }^{41,46}$ It is only briefly presented here.

The finite-size chain of ferromagnetically coupled local spins is described by the magnetic Hamiltonian, $H_{M a g}$ :

$$
\begin{aligned}
H_{M a g}= & \sum_{i=1}^{N-1} J \vec{S}_{i} \cdot \vec{S}_{i+1}+\sum_{i}\left[D S_{i, z}^{2}+E\left(S_{i, x}^{2}-S_{i, y}^{2}\right)\right] \\
& +\sum_{i} g \mu_{B} \vec{S}_{i} \cdot \vec{B}
\end{aligned}
$$

where $\vec{S}_{i}$ is the spin of the atom $i$ in the chain, and $J$ is the Heisenberg exchange coupling $(J<0$ for the ferromagnetic chains considered here). Only couplings between first neighbors are included. $D$ and $E$ terms are anisotropy terms (see a discussion of their origin in Ref. 47), $g$ is the gyromagnetic factor, and $\mu_{B}$ is the Bohr magneton. The applied B field is taken along the anisotropy $z$ axis. Two applications are presented here, a pure Heisenberg chain $(D=E=0)$ of local spins $\frac{1}{2}$ (Sec. III) and a chain of local spins 2 with anisotropy (Sec. IV).

The eigenenergies, $E_{j}$, and eigenvectors, $\left|\Phi_{j}\right\rangle$, of the Hamiltonian [Eq. (1)] are obtained by diagonalization in the basis set formed by direct products of eigenstates of $\vec{S}_{i}^{2}$ and $S_{i, z}$, the squared modulus and projections on the $z$ axis of the local spins: $\left|M_{1}, M_{2}, \ldots, M_{N-1}, M_{N}\right\rangle\left(M_{i}\right.$ is an eigenvalue of the $S_{i, z}$ operator). This diagonalization step is performed using a standard library routine for the low $N$ cases discussed here. The magnetic transitions induced by collision of an electron on the chain are described in the strong coupling approximation: The evolution induced by the interaction terms in the magnetic Hamiltonian is slow, much slower than the collision process, so that one can resort to a sudden approximation in which the collision only concerns the electron and one atom in the chain. The scattering amplitude, $T$, is then diagonal in the basis set formed by the eigenstates of $\vec{S}_{T}^{2}$ and $S_{T, z}$ (quantum numbers, $S_{T}$ and $M_{T}$ ), where $\vec{S}_{T}$ is the total spin of the scattering atom + electron system. Only two $S_{T}$ values are possible: $S_{T}=$ $S \pm 1 / 2$. The transition amplitude from a state of the chain $\left|\Phi_{i}\right\rangle$ to a state $\left|\Phi_{f}\right\rangle$ induced by collision with an electron (the projection of the electron spin on the quantization $z$ axis changes from $m_{i}$ to $\left.m_{f}\right), A_{i, m_{i} \rightarrow f, m_{f}}$, is then written as:

$$
A_{i, m_{i} \rightarrow f, m_{f}}=\sum_{M_{T}, S_{T}}\left\langle m_{f}, \Phi_{f} \mid S_{T}, M_{T}\right\rangle T^{S_{T}}\left\langle S_{T}, M_{T} \mid m_{i}, \Phi_{i}\right\rangle,
$$

where $T^{S_{T}}$ is the amplitude for the transmission of the electron in the symmetry $S_{T}$. Note that this amplitude only contains spin coupling matrix elements and the $T^{S_{T}}$ amplitudes. It can correspond to a tunneling process (electrons transmitted from tip to substrate) or to a scattering process (substrate electrons scattered by one atom of the chain). Note that this formalism is equivalent to that commonly used to treat rotational excitation of molecules (free or adsorbed) ${ }^{48-50}$ by electrons, where the orbital angular momentum plays a role analogous to that of the spin angular momentum.

\section{A. Excitation by tunneling electrons}

Let us first consider the case of the excitation of the chain by a tunneling electron. In the various systems on which we performed an ab initio study of the tip-substrate transmission, ${ }^{34,44}$ we found that only one symmetry $S_{T}$ is active, so that Eq. (2) simplifies into the simple product of a global flux factor $\left|T^{S_{T}}\right|^{2}$ and a spin coupling factor that depends on the initial and final states. From this, one gets the probabilities for the various excitations of the chain from state $\left|\Phi_{i}\right\rangle$, induced by an electron tunneling through one atom in the chain:

$$
\begin{aligned}
& P_{E x c}(i \rightarrow f) \\
& \quad=\sum_{m_{i}, m_{f}}\left|\sum_{M_{T}}\left\langle m_{f}, \Phi_{f} \mid S_{T}, M_{T}\right\rangle\left\langle S_{T}, M_{T} \mid m_{i}, \Phi_{i}\right\rangle\right|^{2},
\end{aligned}
$$

where the sums over $m_{i}$ and $m_{f}$ depend on the conditions of the polarization of the tip and substrate. The normalization of the probabilities in Eq. (3) is the following: for nonpolarized tip and substrate, for a given $i$, the sum including both values of $S_{T}$ is equal to 2 (the spin degeneracy in the incident channel). The projections in Eq. (3) and the similar ones in Sec. II B are evaluated in the basis set built on local spins $\left(\left|M_{1}, M_{2}, \ldots, M_{N-1}, M_{N}\right\rangle\right)$, in which both $S_{\mathrm{T}}$ and $\Phi_{\mathrm{i}}$ states are expressed.

In the applications presented below, we chose two model systems for which we assumed that only one $S_{T}$ symmetry was active in the excitation process. In Sec. III, we consider chains of spins $\frac{1}{2}$ with the tunneling symmetry $S_{T}=0$, and in Sec. IV, we consider chains of spins 2, with the tunneling symmetry $S_{T}=3 / 2$.

\section{B. Lifetime of magnetic excitations}

Let us now consider the de-excitation of an excited state of the chain by scattering of a substrate electron. A chain of magnetic atoms adsorbed on a surface is continuously hit by electrons from the substrate that are scattered back into the substrate. At very low $T$, this collision cannot excite the chain, but it can very efficiently induce de-excitations of excited states of the chain. This decay process can be described as induced by electron-hole pair creation.

The lifetime of an excited magnetic state $\Phi_{i}$ of the chain, $\tau_{i}$ (inverse of its total decay rate, $\Gamma_{T o t, i}$ ), can be expressed for a vanishing temperature from the $T$ transition matrix for electron scattering by one of the atoms in the chain as (see 
discussion in Refs. 41,46,51):

$$
\begin{aligned}
\frac{1}{\tau_{i}}= & \Gamma_{T o t, i}=\sum_{f} \Gamma_{i, f}= \\
= & \sum_{f} \frac{2 \pi \delta \Omega_{f}}{\hbar} \sum_{k_{i}, k_{f}, m_{i}, m_{f}}\left|\left\langle k_{f}, m_{f}, \Phi_{f}|T| k_{i}, m_{i}, \Phi_{i}\right\rangle\right|^{2} \\
& \times \delta\left(\varepsilon_{i}-\varepsilon_{f}\right) \delta\left(\varepsilon_{i}-E_{F}\right),
\end{aligned}
$$

where $\Gamma_{i, f}$ is the partial decay rate of state $i$ towards the lowerlying states $f ; \Phi_{f}$ is the final state of the decay, associated to the energy transfer $\delta \Omega_{f}=E_{i}-\mathrm{E}_{f}$. The total energy is
$E_{T}=E_{i}+\varepsilon_{i}=E_{f}+\varepsilon_{f}$. The initial state and final state of the substrate electrons are labeled by their energy $\varepsilon_{i}$ and $\varepsilon_{f}$, their wave number, $k_{i}$ and $k_{f}$, and by their spin projections on the quantization axis, $m_{i}$ and $m_{f}$. Equation (4) is derived under two assumptions (see details in Refs. 41,46,51): (i) the system temperature is assumed to vanish, and (ii) the $T$ transition matrix elements are assumed to be constant in the small energy interval involved in the decay process. In the strong coupling approach, the $T$ matrix can be expressed as diagonal terms in the tunneling $S_{T}$ symmetries [Eq. (2) above]. The decay rate can then be expressed as:

$$
\begin{aligned}
\frac{1}{\tau_{i}} & =\sum_{f} \frac{2 \pi \delta \Omega_{f}}{\hbar} \sum_{k_{i}, k_{f}, m_{i}, m_{f}} \delta\left(\varepsilon_{i}-\varepsilon_{f}\right) \delta\left(\varepsilon_{i}-E_{F}\right)\left|\sum_{S_{T}}\left\langle k_{f}\left|T^{S_{T}}\right| k_{i}\right\rangle \sum_{M_{T}}\left\langle m_{f}, \Phi_{f} \mid S_{T}, M_{T}\right\rangle\left\langle S_{T}, M_{T} \mid m_{i}, \Phi_{i}\right\rangle\right|^{2} \\
& =\sum_{f} \frac{2 \pi \delta \Omega_{f}}{\hbar} \sum_{k_{i}, k_{f}, m_{i}, m_{f}} \delta\left(\varepsilon_{i}-\varepsilon_{f}\right) \delta\left(\varepsilon_{i}-E_{F}\right)\left|\sum_{S_{T}}\left\langle k_{f}\left|T^{S_{T}}\right| k_{i}\right\rangle A_{i, m_{i} \rightarrow f, m_{f}}^{S_{T}}\right|^{2} .
\end{aligned}
$$

This expression incorporates the two possible values of $S_{T}$ and the corresponding interference effects. It can be further simplified if only one $S_{T}$ symmetry is considered to be active in the de-excitation process. In that case, Eq. (5) reduces to the product of a flux factor and a spin coupling factor. ${ }^{41,43}$ For the present model study, we assumed that the two $S_{T}$ symmetries contribute to the decay, and, similar to Ref. 41, we used a statistical expression neglecting the interference terms between transitions within the two $S_{T}$ symmetries that can be seen in Eq. (5). Finally, the decay rate of state $i$ is obtained as:

$$
\frac{1}{\tau_{i}}=\sum_{f} \Gamma_{i, f}=T_{\text {total }}\left(E_{F}\right) \sum_{f} \frac{\delta \Omega_{f}}{h} P_{\text {Spin }}(i \rightarrow f),
$$

where $T_{\text {total }}\left(E_{F}\right) / h$ is the total electron flux hitting the adsorbate per unit of energy and per unit of time; it appears as a general factor for the decay rate of all magnetic states in the system.

It is given by:

$$
\begin{aligned}
& T_{\text {total }}\left(E_{F}\right) \\
& \quad=\sum_{S_{T}}(2 \pi)^{2} \sum_{k_{i}, k_{f}} \delta\left(\varepsilon_{i}-\varepsilon_{f}\right) \delta\left(\varepsilon_{i}-E_{F}\right)\left|\left\langle k_{f}\left|T^{S_{T}}\right| k_{i}\right\rangle\right|^{2} .
\end{aligned}
$$

$P_{\text {Spin }}(i \rightarrow f)$ is a mean decay efficiency over the two $S_{T}$ symmetries, and it only depends on spin coupling coefficients. The above expression corresponds to decay by scattering of a substrate electron on one given atom in the chain, and contributions for the different atoms have to be added. In the case of an open chain of atoms, these contributions are a priori different for the different sites in the chain via the $P_{\text {Spin }}(i \rightarrow f)$ factor, leading to a global $\bar{P}_{\text {Spin }}(i \rightarrow f)$ factor. From Eq. (6), one can deduce the branching ratio for the decay of state $i$ to a lower-lying state $j$, as:

$$
P_{\text {decay }}(i \rightarrow j)=\frac{\Gamma_{i, j}}{\sum_{f} \Gamma_{i, f}}=\frac{\delta \Omega_{j} \bar{P}_{\text {Spin }}(i \rightarrow j)}{\sum_{f} \delta \Omega_{f} \bar{P}_{\text {Spin }}(i \rightarrow f)}
$$

Note that these branching ratios only depend on the energy defect of the decay and on spin coupling coefficients and not on the absolute strength of the coupling to the substrate governed by $T_{\text {total }}\left(E_{F}\right) / h$.

In the model applications below, we took a numerical value of $T_{\text {total }}\left(E_{F}\right)$ equal to 1 , similar to what we found in the case of single $\mathrm{Mn}$ adsorbates on $\mathrm{CuN} / \mathrm{Cu}(100)$ surfaces. ${ }^{41}$ It should be representative of magnetic adsorbates separated from a metal surface by an ultrathin insulator layer and thus only weakly coupled to the substrate. In the case of magnetic atoms directly adsorbed on a metal surface, the interaction with the substrate electrons is much stronger, leading to much shorter lifetimes ${ }^{35,52-54}$ and possibly to different behaviors. ${ }^{55}$

\section{Rate equation for the population dynamics}

To describe the dynamics of the magnetization of a ferromagnetic chain, we used a semiclassical rate equation approach. It consists in describing the evolution of the population of the different states of the chain as functions of time, $t$, due to both the relaxation of the excited state's population via electron-hole pair creation and/or excitation by tunneling electrons. This approach has been very successfully applied in several studies of magnetic state dynamics. ${ }^{38,41,56,57}$ Basically, it only considers populations and thus neglects all kinds of coherence effects between the various decays or excitations by successive electrons. The time evolution of the populations, $\operatorname{Pop}_{i}(t)$, of the different states $i$ due to relaxation is given by the rate equation:

$$
\frac{d \operatorname{Pop}_{i}(t)}{d t}=-\operatorname{Pop}_{i}(t)\left(\sum_{j \neq i} \Gamma_{i, j}\right)+\sum_{j \neq i} \operatorname{Pop}_{j}(t) \Gamma_{j, i},
$$

where $\Gamma_{i, j}$ is the partial decay rate of state $i$ towards state $j$ (Sec. II B). Equation (9) is solved numerically using an exponential approximation of the population decay for finite 
time steps. In the applications below, the effect of tunneling electrons was modeled as the periodic injection of an electron in the junction. This does not represent a real random flow of electrons but has the advantage of being very efficient in visualizing the effect of relaxation and of tunneling electrons separately. The instantaneous injection of an electron in the junction at time $t$ leads to a sudden change in the various populations, $\triangle P$ op $_{i}$ equal to:

$$
\begin{aligned}
\Delta \operatorname{Pop}_{i}= & -\operatorname{Pop}_{i}(t) \sum_{j \neq i} C P_{E x c}(i \rightarrow j) \\
& +\sum_{j \neq i} \operatorname{Pop}_{j}(t) C P_{E x c}(j \rightarrow i),
\end{aligned}
$$

where $P_{E x c}(i \rightarrow j)$ is given by Eq. (3), and $\mathrm{C}$ is a normalization constant.

In the applications below, we chose to mimic the situation of an STM tip located above an atom in the chain, at constant height, with a constant bias; as a result of the magnetic field sudden reversal at $t=0$, the system is initially in the upper state of the $S_{T o t}=N / 2$ manifold, i.e., only one state is populated. The constant coefficient $\mathrm{C}$ in Eq. (10) is chosen such that Eq. (10) corresponds to the injection of a single electron, at time $t=0$. The number of injected electrons then varies along the relaxation according to the variation of the magnetic state of the chain, because of the variation of the junction conductance with the population of the various states of the chain (see Sec. III D) and similar effects due to excited state population in Refs. 38,41,56,57).

\section{FINITE-SIZE HEISENBERG CHAIN}

In a first step, we consider the simple case of a chain of $N$ spins $1 / 2$ with a Heisenberg exchange coupling between first neighbors. The local spin at site $i$ is $\vec{S}_{i}$, and its projection on the quantization $z$ axis is $S_{i z}$. The magnetic Hamiltonian of the system in the presence of an applied magnetic field, $\vec{B}$, along the $z$ axis, is:

$$
H_{M a g}=\sum_{i=1}^{N-1} J \vec{S}_{i} \vec{S}_{i+1}+\sum_{i=1}^{N} g \mu_{B} S_{i z} B_{z},
$$

where $g$ is the gyromagnetic factor, and $\mu_{B}$ is the Bohr magneton. The total spin of the chain is given by: $\vec{S}_{T o t}=\sum_{i} \vec{S}_{i}$. In the numerical application below, we took a $g$ factor equal to 2 and an Heisenberg coupling equal to $6 \mathrm{meV}$. In the absence of a B field, the ground state of the system is the degenerate state of maximum total spin: $S_{T o t}=N / 2$ (quantum numbers $\left.M_{T o t}=S_{T o t, z}\right)$. In a finite field, the ground state splits in $N+$ 1 components according to the Zeeman effect. The magnetic switch, i.e., the reversal of the magnetic field direction, thus leads to the inversion of the order of the sublevels inside the $S_{T o t}$ subspace; the instantaneous reversal of the B field leads to the population of the highest sublevel of the Zeeman structure, which then relaxes down to the lowest sublevel, i.e., to the relaxation from $M_{T o t}=N / 2$ to the $M_{T o t}=-N / 2$ sublevel. For a chain adsorbed on a surface, the relaxation occurs by electron-hole pair creation with the selection rule $\Delta M_{T o t}=$ \pm 1 , i.e., the population cascades down the Zeeman structure with $\Delta M_{T o t}=-1$ jumps. Higher-lying states with other values of the $S_{T o t}$ are not involved at $0 \mathrm{~K}$, for a low applied field. ${ }^{2}$ One can then view the magnetic switch in this case as the global quantum rotation of the total spin of the chain in $2 \mathrm{~N}$ quantum jumps, exactly corresponding to the Néel-Brown view. It also corresponds to the case studied recently by Wang and Sham; ${ }^{58}$ a stochastic Schrödinger equation approach allowed them to follow the quantuml trajectory of the magnetization and discuss the associated noise.

\section{A. Magnetization switch induced by interaction with the substrate}

The method outlined in Sec. II enables us to evaluate the rate for the various jumps and thus to describe the time dependence of the magnetization of the chain as a function of time following the sudden B field reversal for a vanishing temperature. Figure 1 presents the time dependence of the various sublevels in the $S_{T o t}=N / 2$ subspace in the case of a chain of $N=12$ atoms and an applied B field of $0.1 \mathrm{~T}$. The flux factor $T(E)$ (see Sec. II) is taken equal to 1 , so that the timescale is characteristic of a spin chain adsorbed on an ultrathin insulator on a metal substrate (see a discussion in the review Ref. 43). At time $t=0$, the system is in the highest level $\left(M_{T o t}=+6\right)$, and the corresponding population decreases exponentially, leading to a population of all the sublevels and ultimately to the only population of the ground state $\left(M_{T o t}=-6\right)$. This results in effective time delays between the various states in the manifold; they appear and disappear successively (the complete magnetization switch requires $N$ transitions, i.e., 12 transitions in the case of Fig. 1). The ground state is not populated immediately after the B reversal; it starts to be populated only after around $3 \mathrm{~ns}$ in Fig. 1. However, one can notice that several states are populated at the same time; typically around $4 \mathrm{~ns}$, all states are populated significantly,

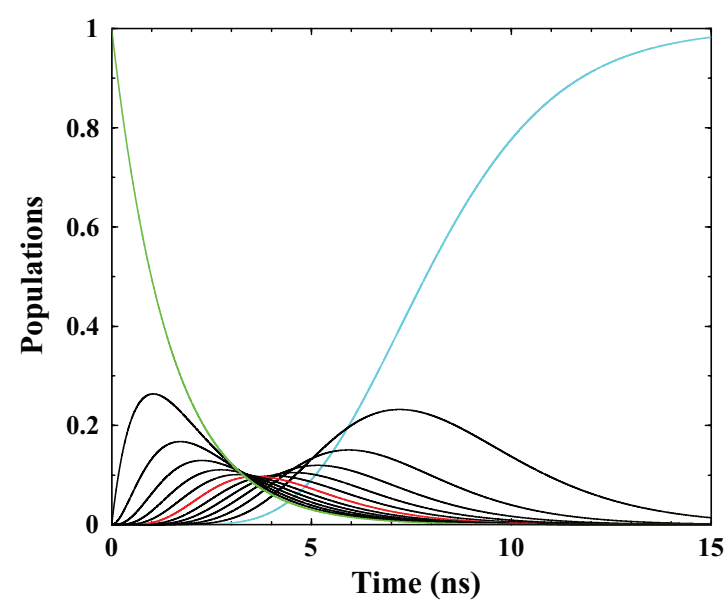

FIG. 1. (Color online) Populations of the 13 sublevels of the $S_{\text {Tot }}=6$ lowest manifold of a chain of 12 atoms as a function of time following the reversal of the direction of the applied B field $(0.1 \mathrm{~T})$. The system is initially in the highest state of the manifold $\left(M_{T o t}=+6\right.$, full green line $)$ and relaxes to the ground state $\left(M_{T o t}=\right.$ -6 , full cyan line). The populations of the various $M_{T o t}$ states appear and disappear successively. The population of the central state of the manifold $\left(M_{T o t}=0\right)$ is shown in red. 
and it is only in the early and late times that only one state is populated.

\section{B. Dependence of the magnetization switch time on the chain length (long chains)}

We studied the variation of the magnetization dynamics with the length of the spin chain numerically following Sec. II for short chains $(N<16)$. For longer chains, the number of states to be considered becomes excessively large, and we switched to an analytical approach only considering the process at low field and thus only computing the transitions among the sublevels of the ground manifold, $S_{T o t}=N / 2$. Let us consider one of these sublevels, with $M_{T o t}=N / 2-j$. Its decay by collision with the substrate electrons only populates the sublevel just below in the manifold, i.e., $M_{T o t}=N / 2-$ $j-1$. Considering the spin coupling element at play and the fact that the energy associated with all these individual decays is equal to $\Delta E=g \mu_{B} B$, we find the following decay rate for the $M_{\text {Tot }}=N / 2-j$ sublevel:

$$
\begin{aligned}
\Gamma\left(M_{T o t}=N / 2-j\right) \\
\quad=\left[\frac{T(E) \Delta E}{8 \pi}\right] \frac{(j+1)(N-j)}{N}, j=0, N .
\end{aligned}
$$

The term between brackets is the decay rate of the highest member of the manifold ( $j=0)$, and the second term yields the dependence of the decay rate along the manifold. The rate maximizes at the center of the manifold. One recognizes that, via the $\Delta E$ term, the decay rate is proportional to $\mathrm{B}$, so that the switch should speed up with increasing applied B. Actually, the only factors that are specific to a given system in Eq. (12) are the $T(E)$ flux factor, $g$ the gyromagnetic factor, and the applied field B. In particular, all these decay rates are independent of the Heisenberg coupling, $J$, so that the behavior defined in Eq. (12) is rather general.

The lower panel of Fig. 2 presents the population of the ground state of the system for various chain lengths as a function of time after the reversal of the $\mathrm{B}$ direction $(\mathrm{B}=$ $0.1 \mathrm{~T})$. The time for a magnetization switch increases with the length of the chain. Actually, the various population curves appear to be roughly translated one from the other so that the increase of the magnetization switch time looks like an increased time delay for the rise of the ground-state population. All this is not so surprising since complete switching requires a number of transitions equal to $N$; however, the decay rate of each individual state depends on the chain length, so the global behavior was not obvious. The upper panel of Fig. 2 presents the magnetization switch in another way; it presents the average magnetic moment of the chain (mean value of the projection of $\vec{S}_{T o t}$ on the B field axis, $\langle\mathrm{M}\rangle$ ), normalized to its maximum value as a function of time. It goes from +1 (highest state) at the time of the $\mathrm{B}$ reversal to -1 (ground state) for asymptotically long times. From Fig. 2, one can define a switching time in different ways; one can define the time, $T(\langle\mathrm{M}\rangle=0)$, at which the chain magnetic moment is zero, which could be thought of as a half switch. One can also look at the time, $T(90 \%)$, at which the population of the ground state is equal to $90 \%$, i.e., when the switch is almost complete. Figure 3 presents these two "switching times" $(2 T[\langle\mathrm{M}\rangle=0]$ and $T[90 \%])$ as a function of the number of atoms in the chain

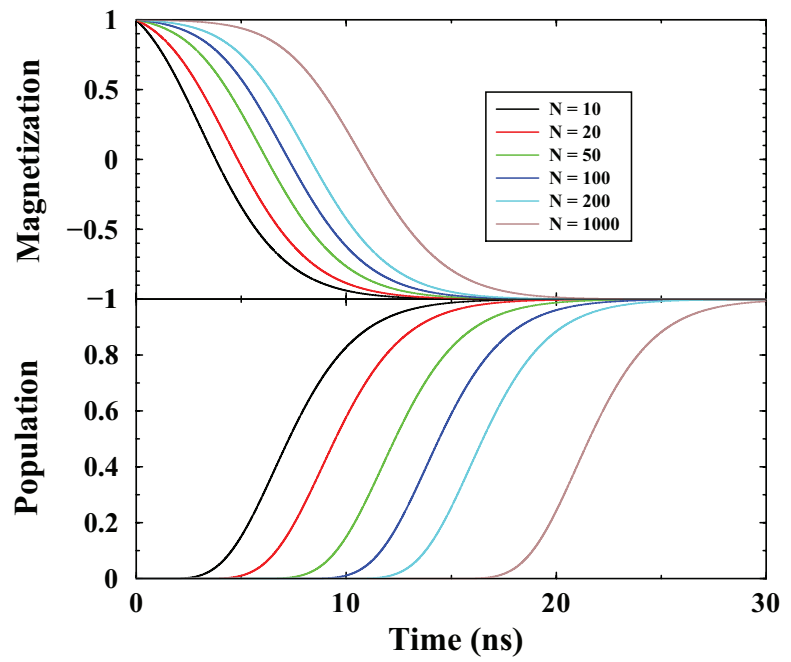

FIG. 2. (Color online) Upper panel: variation of the magnetic moment of a chain of $N$ atoms as a function of the time following the reversal of the direction of the applied magnetic field $(0.1 \mathrm{~T})$. The magnetic moment is plotted relative to its maximum value. The number of atoms in the chain is given in the insert (the shorter the chain, the faster the magnetic switch). Lower panel: variation of the population of the ground state of a chain of $N$ atoms as a function of the time following the reversal of the direction of the applied magnetic field $(0.1 \mathrm{~T})$. Same color code as in the upper panel (the shorter the chain, the faster the magnetic switch).

(field $\mathrm{B}=0.1 \mathrm{~T}$ ). It appears that the two switching times, though numerically different as expected, exhibit the same logarithmic behavior as a function of $N$, for large enough $N$.

At this point, one can stress that the decay rate given by Eq. (12) is actually obtained by only considering the wave functions of the $S_{T o t}=N / 2$ manifold and the energy difference between two neighboring states in the manifold. The $T(E)$ flux factor appears as a global factor. So any ensemble of spins

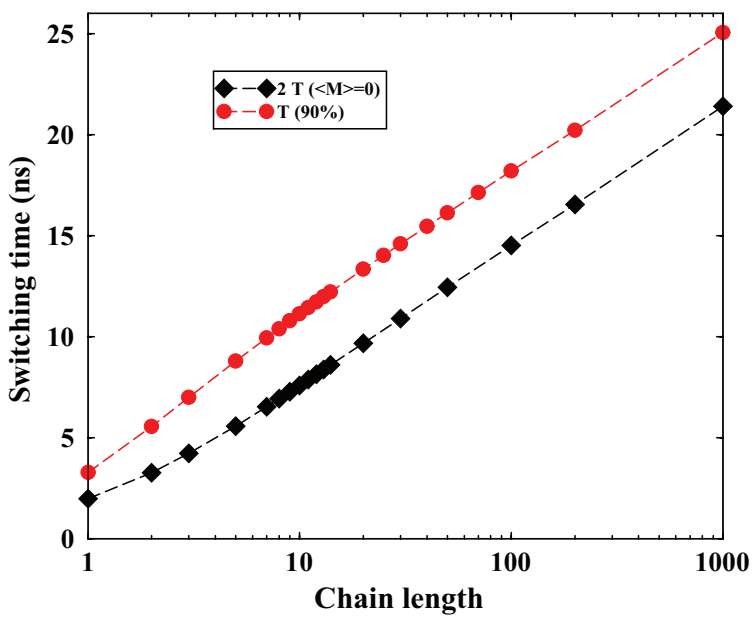

FIG. 3. (Color online) Switching time of the magnetization of a chain of atoms when the direction of the applied B field $(0.1 \mathrm{~T})$ is reversed, as a function of the chain length (number of atoms in the chain). Two definitions of the switching time are shown: twice the time for the magnetic moment of the chain (projection on the field axis) to reach zero $(2 T[\langle\mathrm{M}\rangle=0])$ and the time for the population of the ground state to reach $90 \%, T(90 \%)$. 
$\frac{1}{2}$, coupled by an Heisenberg Hamiltonian with a ground-state manifold corresponding to $S_{T o t}=N / 2$, will behave in the same way, and the dynamics under the reversal of an applied B field will be the same, irrespective of its actual structure (open chain or ring) and dimensionality (one-dimensional [1D] chain or two-dimensional [2D] adsorbed island). In particular the log $N$ behavior of the switching time (global rotation) should be a general feature of the Heisenberg systems.

\section{Magnetization switching at high magnetic field}

A change of the strength of the B field in the low-field range only leads to a scaling effect on the time behavior. Indeed, as discussed in Sec. II and seen in Eq. (12), the decay rates of the various states are obtained as products of a term only depending on spin couplings and of an energy difference term that is proportional to $\mathrm{B}$. As a consequence, when B is changed, the time dependencies in Fig. 1 are identical if the time is scaled proportionally to $\mathrm{B}$ (the larger $\mathrm{B}$, the faster the time evolution). For the $N=12$ chain, this remains true as long as the B field is small enough for the decay to only concern the sublevels of the $S_{T o t}=6$ subspace. However, for very large B, the higher-lying $S_{T o t}=6, M_{T o t}=6$ sublevel crosses levels of higher-lying manifolds (different values of $S_{T o t}$ ), opening new decay routes for the higher-lying states and thus shortening the magnetic switch time. This is illustrated in Fig. 4, which presents the population of the ground state of an $N=12$ chain as a function of a scaled time for various values of the applied $B$ field. The scaled time is equal to the product of the physical time (in ns) and the applied B field (in tesla). At low fields, all the curves in Fig. 4 fall on each other and are represented by the single $0.1 \mathrm{~T}$ curve. As B is increased, the magnetic switch becomes faster in scaled time above a certain threshold; in Fig. 4, the curve $\mathrm{B}=2 \mathrm{~T}$ is slightly above the threshold and is

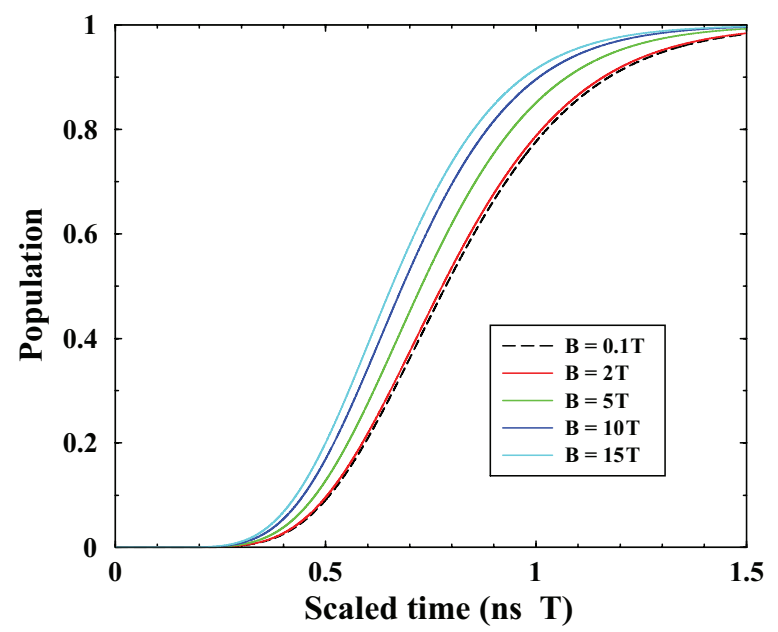

FIG. 4. (Color online) Population of the ground state of a chain of 12 atoms as a function of a scaled time following the reversal of the applied B field direction. The scaled time is equal to the product of the physical time in nanoseconds and the field strength expressed in Tesla. Various strengths of the B field are shown (see insert). At low fields, all the population curves are superimposed and represented by the dashed line, which shows the results for $0.1 \mathrm{~T}$; as the B field is increased, the ground-state population increases earlier. associated with a tiny shortening of the magnetization switch; the larger B field cases (10 and $15 \mathrm{~T}$ ) exhibit a very clear shortening. One can stress that the threshold for this effect is linked to a comparison between the Heisenberg structure and the Zeeman structure, and as such, its value depends on the exchange coupling, $J$, as well as on the precise structure of the system (chain or ring); this is at variance with the time dependence of the decay at low field (Figs. 1 and 2), which is completely independent of the strength of the ferromagnetic Heisenberg coupling. The new decay route appears when states in the ground manifold $\left(S_{T o t}=6\right)$ cross states from a higher manifold $\left(S_{T o t}=5\right)$; then the selection rules for a transition induced by an electron $\left(\Delta S_{T o t}=0, \pm 1 ; \Delta M_{T o t}=0, \pm 1\right)$ can be fulfilled, and the population cascade a priori also involves states from the $\left(S_{T o t}=5\right)$ manifold. The lowest states in the $S_{T o t}=5$ manifold correspond to spin waves quantized in the finite-size chain. As discussed in Ref. 45, tunneling electrons are very efficient in exciting spin waves in a Heisenberg spin chain, and furthermore the spin waves are the only states excited by tunneling electrons; similarly, in the present study, substrate electrons are very efficient in pumping the population from the ground manifold to the spin waves. The shortening of the magnetization time switch seen in Fig. 4 then corresponds to the combined action of two switch mechanisms, both induced by collisions with electrons from the substrate: the global rotation of the ferromagnet and the decay via spin wave excitation.

\section{Magnetization switch induced by injected electrons}

The above discussion of the effect of the strength of the magnetic field (Fig. 4) stresses the efficiency of the spin waves for accelerating a magnetization switching. This phenomenon can be further put forward by considering the action of tunneling electrons injected from an STM (scanning tunneling microscope) tip into one of the atoms in the chain. To mimic the effect of a current of tunneling electrons, we studied the magnetization switching of a chain of atoms when an electron is injected periodically (constant time interval $d t$ ) into one of the atoms of the chain. The injected electron is supposed to be fully polarized in the spin down state with respect to the quantization axis. This simple scheme neglects the random aspect of electron tunneling. However, it should carry the main physical characteristics of the electron-induced magnetization switch; in addition, it allows easy visualization of the relative role of relaxation by electron-hole pair creation and excitation by tunneling electrons. The evolution of the populations of the magnetic states in the chain is then governed by two aspects: relaxation inside the manifold $S_{T o t}=N / 2$ induced by collisions with the substrate electrons (see above sections), and periodic kicks induced by the tunneling electrons. The STM bias is chosen to be larger than all the excitation thresholds. In the case of a ferromagnetic chain, excitation by tunneling electrons from states in the $S_{T o t}=N / 2$ manifold only leads to spin-wave excitations that relax very quickly to the lower $S_{T o t}=N / 2$ manifold, at least on the timescale of the magnetization switching (see Refs. 45 and 46 for a discussion of the excitation of ferromagnetic chains by tunneling electrons and of the excited state lifetimes). Excitations from the spin 


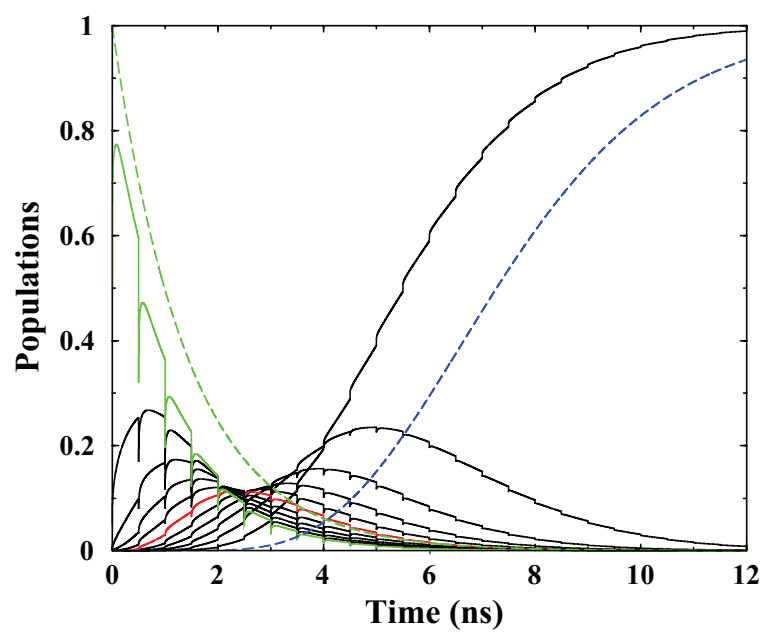

FIG. 5. (Color online) Populations of the 11 sublevels of the $S_{T o t}=5$ lowest manifold of a chain of 10 atoms as a function of time following the reversal of the direction of the applied B field $(0.1 \mathrm{~T})$. The system is initially in the highest state of the manifold $\left(M_{T o t}=\right.$ $+5)$ and relaxes to the ground state $\left(M_{T o t}=-5\right)$. An electron from an STM tip with spin polarization down is injected into an end atom of the chain at intervals of $0.5 \mathrm{~ns}$, creating the sharp structures in the populations. The populations of the various $M_{T o t}$ states (full lines) appear and disappear successively. The population of the initial upper state of the manifold $\left(M_{T o t}=5\right)$ is shown by a full green line, and that of the central state of the manifold $\left(M_{T o t}=0\right)$ is shown by a full red line. The dashed curves (green and blue) correspond to the case where no electron is injected (populations of the upper and lower states of the manifold, respectively).

waves to further higher-lying states are also possible, but they only play a role for the shortest $d t$ considered here.

Figure 5 presents the time evolution of the populations of the 11 states from the $S_{T o t}=5$ manifold of a chain of 10 atoms as a function of time following a reversal of the B field direction $(\mathrm{B}=0.1 \mathrm{~T})$. An electron is injected into an end atom of the chain periodically ( $d t=0.5 \mathrm{~ns}$ ), starting at time $t=0$. At the time of the injection of the tunneling electron, a sharp change of the population of all the states in the manifold is visible, corresponding to the inelastic effect of the tunneling electrons [Eq. (10)]; most of these changes are sharp drops due to the excitation toward spin waves. For example, the sharp drop at $t=0.5 \mathrm{~ns}$ in the population of the $M_{T o t}=$ 5 state in the $S_{T o t}=5$ manifold (full green line) is due to excitation of higher-lying spin waves. The population in the spin waves relaxes very quickly, and shortly after the drop, the $M_{T o t}=5$ state recovers some population from the decaying spin waves. Actually, for the present tunneling electron range, there are clearly two timescales in the magnetization dynamics: a short timescale associated with the spin waves, and a longer timescale associated with the global rotation of the $S_{T o t}=5$ states of the chain.

Globally, the effect of the injected electrons is to accelerate the magnetization switching. This is visible in Fig. 5, which shows the population of the highest and lowest states in the $S_{\text {Tot }}=5$ manifold (dashed lines) in the absence of tunneling electrons. One can notice that the effect of the tunneling electrons on the population of the ground state is an increase both via decay of the spin waves and via transitions induced by tunneling electrons inside the $S_{T o t}=5$ manifold. At this point, one can stress that the assumption of fully polarized tunneling electrons restricts the number of possible transitions induced by the tunneling electrons. The calculation was normalized to describe the situation of a constant tip height; as a consequence, at $t=0$, spin-down electrons collide on a spin-up chain and are very efficiently transmitted (the equivalent current in Fig. 5 is initially $0.32 \mathrm{nA}$ ); in contrast, at very large times, spin-down electrons collide on a spin-down chain, and, with the assumption of the tunneling symmetry (see Sec. II), the transmission probability is very small and vanishes asymptotically. This choice of normalization leads to a tunneling current going to zero as the magnetization switching proceeds and allows us to define the total number of electrons injected in the junction during the switch (see below).

Figure 6 presents the effect of varying the intensity of the current injected into the chain; this is realized by varying the time interval, $d t$, between two successive electron injections. Figure 6 presents the average magnetic moment of the chain $(N=10)$ as a function of time, following the reversal of the applied field $\mathrm{B}(\mathrm{B}=0.1 \mathrm{~T})$. It appears that electron injection is very effective in speeding up the magnetization switching. So, even if the electron injection directly results in the population of states lying higher than those in the lowest $S_{T o t}=5$ manifold, finally it results in a faster population of the ground state, i.e., in a faster magnetization switching. Spin-wave excitation is then a very efficient process for inducing a magnetization switching. If the injected current is strong enough, it beats the effect of spontaneous relaxation (global rotation of the chain magnet induced by substrate electrons), and the switching time becomes very small. The results in Figs. 5 and 6 are obtained for electrons injected into end atoms of the chain. Actually,

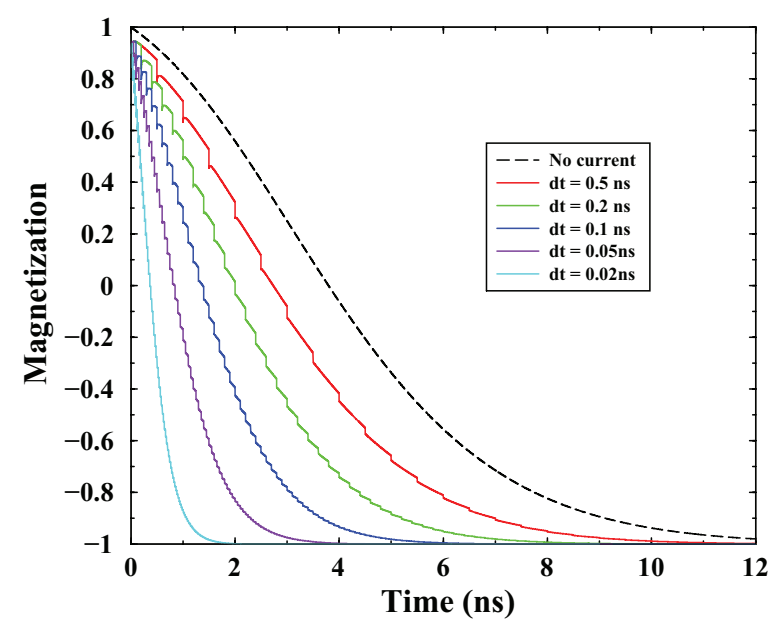

FIG. 6. (Color online) Magnetization (relative mean magnetic moment of the chain) as a function of time following the reversal of the applied B field ( $\mathrm{B}=0.1 \mathrm{~T})$ for an $N=10$ chain in which polarized electrons are injected into an end atom of the chain. The electrons are injected periodically with a constant $d t$ time interval. The different full lines correspond to different $d t$ intervals (see insert): the shorter the $d t$, the faster the magnetization change. The dashed line corresponds to the case where no electrons are injected, and the relaxation only proceeds via collisions with the substrate electrons. 


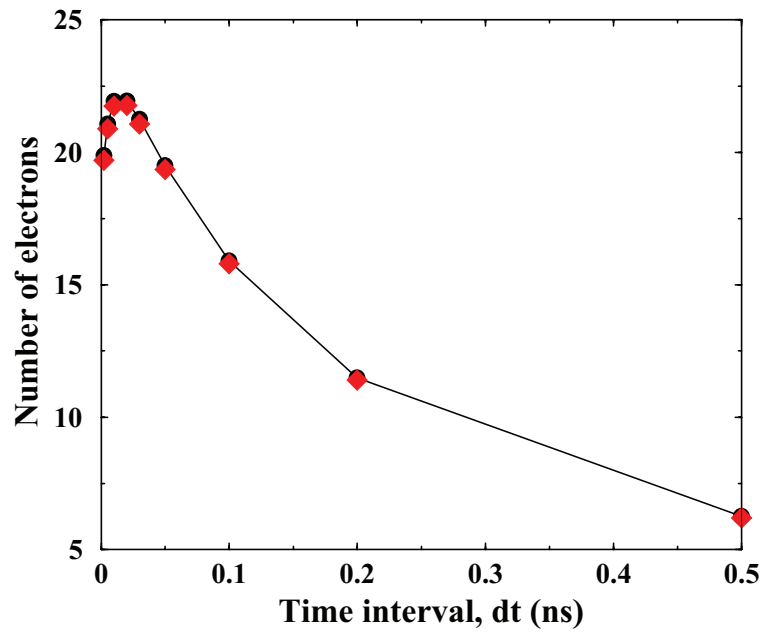

FIG. 7. (Color online) Total number of injected electrons required to perform the magnetization switch as a function of the time interval, $d t$, between injected electrons. The chain contains 10 atoms, and the B field is $0.1 \mathrm{~T}$. Full black circles and full black line: number of electrons for the ground-state population to reach 99\%. Full red diamonds: number of electrons for the ground-state population to reach $90 \%$.

injecting electrons into other atoms in the $N=10$ chain leads to a very small variation of the switching dynamics. Typically for $N=10, \mathrm{~B}=0.1 \mathrm{~T}, d t=0.2 \mathrm{~ns}$, the time $T(90 \%)$ varies by less than one percent when varying the injected atom.

As discussed above, the normalization used for the injected electrons allows the total number of tunnel electrons involved in the magnetization switch to be defined. This total number is presented in Fig. 7 for an $N=10$ chain as a function of the time interval, $d t$. Two definitions of the switch were used (ground-state population equal to $90 \%$ or $99 \%$ ) and lead to almost the same number of injected electrons. At very large $d t$, the total number of electrons injected during the spontaneous relaxation of the chain magnet is very small, and, coherently, the spin-wave excitation does not play a role. As $d t$ decreases, the spin-wave excitation role increases to dominate at small $d t$. There, the total number of electrons required to achieve the magnetization reversal maximizes around 22. This means that the maximum angular momentum that the electrons bring to the chain is equal to $22 \hbar$ (if each injected electron undergoes a spin flip), whereas the complete flip of the chain magnet involves $10 \hbar$. This confirms the very large efficiency of the spin-wave excitation mechanism for magnetization switching; actually, the electron pulse required to flip the chain is very small. This extreme efficiency is partially linked to the particular case studied here (chain of spins $\frac{1}{2}$ excited via $S_{T}=0$ symmetry); it would change when changing $S$ or $S_{T}$, but it would remain very large. From the microscopic quantum study, one can then conclude the extreme efficiency of polarized tunneling electrons in inducing magnetic reversal via the spin-transfer torque and spin-wave excitation.

Magnetization reversal induced by tunneling current (tunneling current-induced changes in the thermal magnetization reversal) has been evidenced in several experimental studies, ${ }^{20-23}$ and the importance of spin-transfer torque in this case has been put forward. The previous discussion was based at $0 \mathrm{~K}$. However, the processes discussed for tunneling electrons should play a role in the case of thermal processes; indeed, substrate electrons above a certain energy can excite spin waves in the chain and lead to reversal, similar to tunneling electrons. The corresponding process is thermally activated, with the activation energy equal to the excitation energy of the spin wave. Spin-wave excitation has already been shown to play a significant role in magnetic reversal in several theoretical works using classical approaches. ${ }^{9,15,16}$

Finally, one can also examine the effect of injecting electrons with a nonpolarized spin. In that case, for asymptotically long times, the system is not entirely in the ground state. Indeed, injected spin-up electrons are able to pump the system off the ground state, and the final asymptotic limit is the result of a dynamical equilibrium between the relaxation induced by the substrate electrons and the pump up and down of the population induced by the injected electrons. This is illustrated in Fig. 8, which presents the time evolution of the magnetization of an $N=10$ chain (mean magnetic moment of the chain relative to its maximum value) following the reversal of the applied magnetic field direction $(\mathrm{B}=0.1 \mathrm{~T})$. Three results are presented: polarized and nonpolarized electrons injected periodically ( $d t=0.2 \mathrm{~ns}$ ), and no injected electrons. At very early times, the effect of polarized and nonpolarized electrons is the same; indeed, only the spin-down electrons are transmitted through the spin-up chain, within the present model system (tunneling symmetry $S_{T}=0$ ), and so at early times, nonpolarized electrons are also efficiently speeding up the magnetization switch. This is at variance with processes at late times, where the chain mean magnetic moment does not go to the -5 limit of a perfectly polarized chain. At late times, every $0.2 \mathrm{~ns}$, nonpolarized electrons are still able to tunnel through and excite the chain (both spin waves and intra- $S_{T o t}=5$ manifold transitions), resulting in the periodic

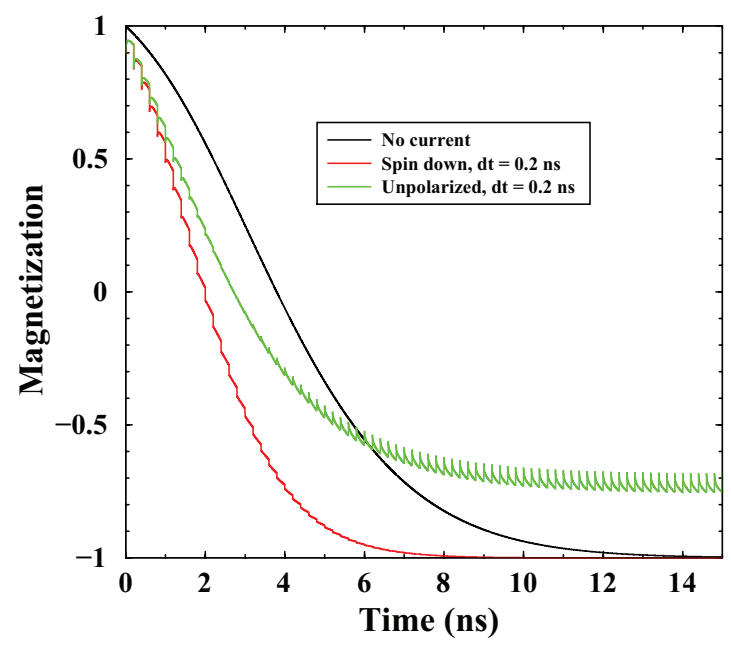

FIG. 8. (Color online) Magnetization (relative mean magnetic moment of the chain) as a function of time following the reversal of the applied B field $(\mathrm{B}=0.1 \mathrm{~T})$ for an $N=10$ chain in which electrons are injected into an end atom of the chain. The electrons are injected periodically with a constant $d t=0.2$ ns time interval. Full red line: the injected electrons are spin down. Full green line: the injected electrons are nonpolarized. Full black line: no injected electrons. 
jumps in the magnetization. The asymptotic value of the magnetization depends on the dynamical equilibrium between the spontaneous relaxation process (global rotation of the chain moment) and the excitation/de-excitation process induced by the injected electrons. As the injected current intensity goes up ( $d t$ goes down), the asymptotic relative magnetization moves away from -1 and tends to 0 for extremely large nonpolarized currents. As a conclusion, one can say that injecting nonpolarized electrons into the chain speeds up the dynamics of the magnetization, but with the drawback of leading to a chain that was not fully polarized at the end; a possible trick would be to use short pulses of nonpolarized electrons that would speed up the early relaxation and let the chain reach a full magnetization after the pulse end.

\section{FINITE-SIZE HEISENBERG CHAINS WITH ANISOTROPY}

We will now consider the case of a chain of local spins coupled by ferromagnetic Heisenberg couplings and interacting with an anisotropic environment. The anisotropy corresponds to the $D$ and $E$ terms in Hamiltonian Eq. (1). The longitudinal term, $D$, simply induces a global energy shift of the energies in the basis set formed of eigenvectors of $\vec{S}_{i}^{2}$ and $S_{i, z},\left|M_{1}, M_{2}, \ldots, M_{N-1}, M_{N}\right\rangle$ in the case of a chain of local spins $\frac{1}{2}$. To illustrate the effect of the anisotropy, we then considered chains of local spins 2 . The $D$ anisotropy significantly alters the wave functions of the lowest-lying states of the chain and consequently can also affect the magnetic switching process. In the numerical applications of the effect of the longitudinal anisotropy (Sec. IV A), we considered a gyromagnetic factor equal to 2, a ferromagnetic Heisenberg coupling of $1 \mathrm{meV}$, and an anisotropy coupling $D$ equal to $-1.5 \mathrm{meV}$ (i.e., a strong anisotropy case). Study of the effect of the transverse coupling $E$ (Sec. IV B) is made with a variable $E$ value.

\section{A. Effect of the longitudinal anisotropy $(D \neq 0, E=0)$}

A negative anisotropy $D$ term ('easy axis' case), partly lifts the degeneracy of the ground state of the chain at vanishing B field, though the projection of the total spin of the chain on the anisotropy axis, $S_{T o t, z}$, remains a good quantum number. For an $N$-atom chain, the doubly degenerate ground state corresponds to the $S_{T o t}=2 N$ and $M_{T o t}= \pm 2 N$ states. The low-energy part of the energy spectrum of an $N=5$ chain is shown in Fig. 9 as a function of the applied field B (the B field is along the anisotropy axis of the local spins). Note that the crossings between the various states are real crossings, although the calculation performed with a finite B grid makes them look like avoided crossings. Starting from $\mathrm{B}=0$, one can see that the ground state splits into two states, $M_{T o t}=+10$ and $M_{T o t}=$ -10 . The magnetic switching process then corresponds to the relaxation of the $M_{T o t}=+10$ (green line in Fig. 9) to the ground $M_{T o t}=-10$ state (red dashed line). It appears that the relaxation process via global rotation of the total spin discussed above (successive transitions with $\Delta M_{T o t}=-1$ ) is impossible at $0 \mathrm{~K}$ in the low-field region, since the intermediate states of the relaxation are not located energetically between the initial and final states (between the $M_{T o t}=+10$ and $M_{T o t}=-10$

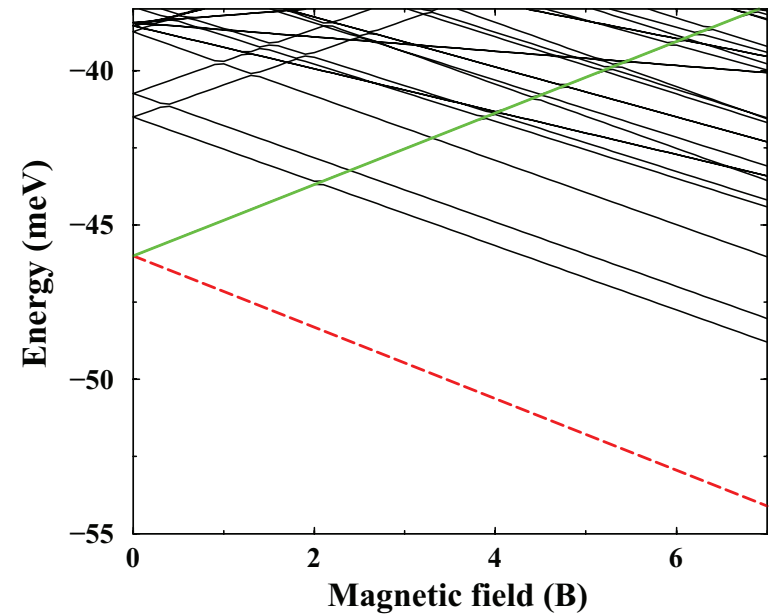

FIG. 9. (Color online) Energy diagram of an $N=5$ chain of $S=$ 2 spins as a function of the applied B field (in Tesla). The local spins are coupled by a ferromagnetic Heisenberg coupling and interact with the environment via an anisotropy $D$ term (easy axis kind). The lowest-lying state energies are shown as full black lines; the energies of the initial and final states of the magnetic switching process $(M=$ +10 and $M=-10$ states) are highlighted in full green and dashed red, respectively.

states). So in the low $\mathrm{B}$ range, the $M_{T o t}=+10$ state is stable; several other states in the spectrum are also stable for the same reason. When the B field is increased, the $M_{\text {Tot }}=+10$ state energy goes up and crosses the entire spectrum to become the highest state for asymptotically large fields. In this limit, the situation discussed in the previous section is recovered, and the magnetic switching can proceed via the global rotation of the total spin. However, for strong anisotropies, like in Fig. 9, this means extremely large B fields. One can see in Fig. 9, that, in the moderate B field range that is shown, the $M_{T o t}=+10$ state only crosses states with negative $M_{\text {Tot }}$ values, whereas the existence of the global rotation mechanism necessitates the presence of the whole spectrum of $M_{\text {Tot }}$ states below the $M_{T o t}=+10$ state. The threshold for global rotation is given by the crossing between $M_{T o t}=+10$ and $M_{T o t}=+9$ states; it amounts to:

$$
B_{T h}=\frac{|D|(2 S-1)}{g \mu_{B}} .
$$

For the case depicted in Fig. 9, the threshold is at 38.9 T, a very high field, due to the strong anisotropy. Just above threshold, the first step of the global rotation is very slow due to the energy defect factor in the transition rate [Eq. (6)].

In a classical view, the magnetic anisotropy generates a potential barrier separating the two opposite magnetizations that the system has to overcome during the reversal, either by a large $\mathrm{B}$ field or by thermal excitation. The existence of a magnetic field threshold for reversal of a magnetic island in the presence of anisotropy has been studied experimentally in detail in Ref. 10; it was analyzed in a classical context ${ }^{10}$ with a field threshold equal to $\frac{2 K}{\mu}$ in the $T=0 \mathrm{~K}$ limit ( $\mu$ is the magnetic moment per atom equal to $g \mu_{\mathrm{B}} S$ with the present notations). $K$ is the anisotropy energy per atom, i.e., the energy barrier to reversal for a single atom, equal to $\frac{|D| S}{2}(2 S-1)$ with the present notations. The classical B threshold is then 


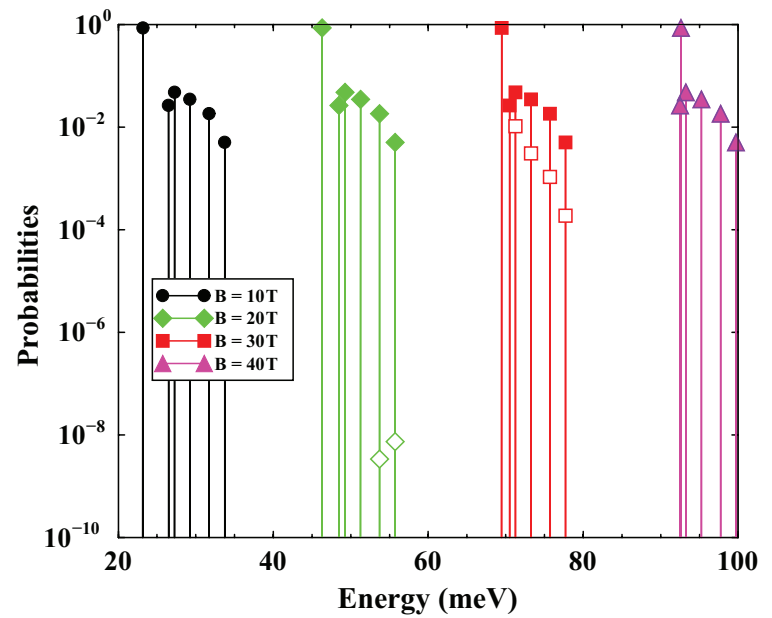

FIG. 10. (Color online) Magnetic switching induced by tunneling electrons in a ferromagnetic chain of 5 local spins $S=2$, with a longitudinal anisotropy. The full symbols present the excitation probability from the initial $M_{T o t}=+10$ state to an excited state $j$ as a function of the excited state energy for various strengths of the applied B field (see insert). The open symbols present the probability for the magnetic switch of the chain via the excited state $j$ (transition from the $M_{T o t}=+10$ state through the intermediate excited $j$ state to the final $M_{\text {Tot }}=-10$ state). Note that for the highest field $(40 \mathrm{~T})$, open and full symbols are superimposed. For the sake of clarity, the energies of the intermediate states $j$ are plotted relative to the energy of the ground state for a vanishing field.

equal to the quantum threshold given by Eq. (13). Note that this threshold is independent of the number of atoms in the magnetic object.

A similar B-threshold effect is visible on the magnetic switch induced by tunneling electrons. Figure 10 presents the excitation probability $P_{E x c}($ init $\rightarrow j$ ) from the initial state (init $\left.: M_{T o t}=+10\right)$ to any excited state $(j)$ and the probability for the magnetic switching induced by a tunneling electron (init $\rightarrow j \rightarrow$ final), where 'final' is the ground state. The latter indirect probability is noted $P_{\text {Ind }}($ init $\rightarrow j \rightarrow$ final). The figure presents these probabilities as a function of energy of the intermediate state, $j$, for several values of the applied field, B. For the sake of clarity, we used for the plot the energies of the states relative to the energy of the ground state for a vanishing field, in order to split the various B's. For B $=10$ $\mathrm{T}$, there is a very strong elastic probability $\left(P_{E x c}[\right.$ init $\rightarrow$ init $]$ ) equal to $13 / 15$ and only five inelastic processes; actually the simple excitation scheme of a pure Heisenberg chain (only quantized spin waves are excited ${ }^{45}$ ) is preserved when a $D$ anisotropy term is included. At $\mathrm{B}=10 \mathrm{~T}$, the indirect probability $P_{\text {Ind }}$ (init $\rightarrow j \rightarrow$ final) is zero, due to the relative energy position of the states; indeed, the only state below the quantized spin waves associated with $M_{T o t}=+9$ and to which they can relax is the $M_{T o t}=+10$ state, so that after an excitation by a tunneling electron, the chain relaxes to the same state. There is a threshold for the indirect process to exist, associated with a crossing between the spin waves and lower $M_{\text {Tot }}$ states. For B $=20 \mathrm{~T}$, one can see in Fig. 10 that the two higher-lying states excited by a tunneling electron can decay to the ground state, though with a very small probability due to the energy defect factor in the decay rate. Note that the B

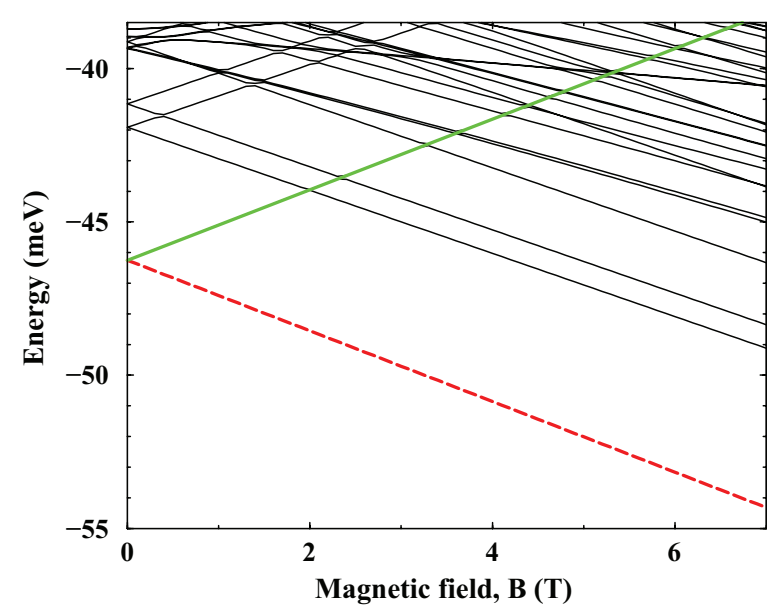

FIG. 11. (Color online) Energy diagram of an $N=5$ chain of $S=2$ spins as a function of the applied B field (in Tesla). The local spins are coupled by a ferromagnetic Heisenberg coupling and interact with the environment via anisotropy $D$ and $E$ terms (easy axis kind). The lowest-lying state energies are shown as full black lines. The full green and dashed red curves that overrun the avoided crossings highlight the energy of the initial and final states of the magnetic switching process $(M \approx+10$ and $M \approx-10$ states $)$.

threshold for the electron-induced processes is similar, though smaller than that for the global rotation process. For $\mathrm{B}=30 \mathrm{~T}$, the indirect probabilities have significantly increased, and for $\mathrm{B}=40 \mathrm{~T}$, the indirect probabilities, $P_{\text {Ind }}($ init $\rightarrow j \rightarrow$ final $)$, are equal to the excitation probabilities, $P_{E x c}($ init $\rightarrow j$ ), i.e., the relaxation always ends up in the ground state (this simply corresponds to the fact that the field is larger than $\mathrm{B}_{T h}$ and the initial $M_{T o t}=+10$ state itself also relaxes to the ground state).

As a conclusion, the longitudinal anisotropy deeply modifies the magnetic switching of the chain: In a low-field region, no spontaneous switch is possible at $0 \mathrm{~K}$; in an intermediate range at finite $\mathrm{B}$, the indirect switch induced by tunneling electrons is possible but with an efficiency much lower than that observed in pure Heisenberg chains; in the large B range (when the Zeeman term dominates over the anisotropy), the situation is comparable to that of the pure Heisenberg chain, with efficient global rotation and switching induced by tunneling electrons.

The above discussion concerns negative $D$ anisotropies. In the case of positive $D$, the ground state of the system at low B is a $M_{T o t}=0$ state, which is insensitive to the direction of the B field direction, so no magnetic switching exists.

\section{B. Effect of a transverse anisotropy term $(D, E \neq 0)$}

The presence of transverse anisotropy further modifies the magnetic switching process. The main change is that, because of $E, M_{T o t}$ is not a good quantum number anymore. So the selection rule on $M_{T o t}$ for electron-induced transitions disappears as well as the stable states at low B discussed in Sec. IV A.

The energies of the low-lying states of a chain of 5 local spins $S=2(J=-1.0 \mathrm{meV}, D=-1.5 \mathrm{meV}, E=0.3 \mathrm{meV})$ are shown in Fig. 11. Though the transverse anisotropy $E$ is significant, the changes in the low-energy part of the spectrum 


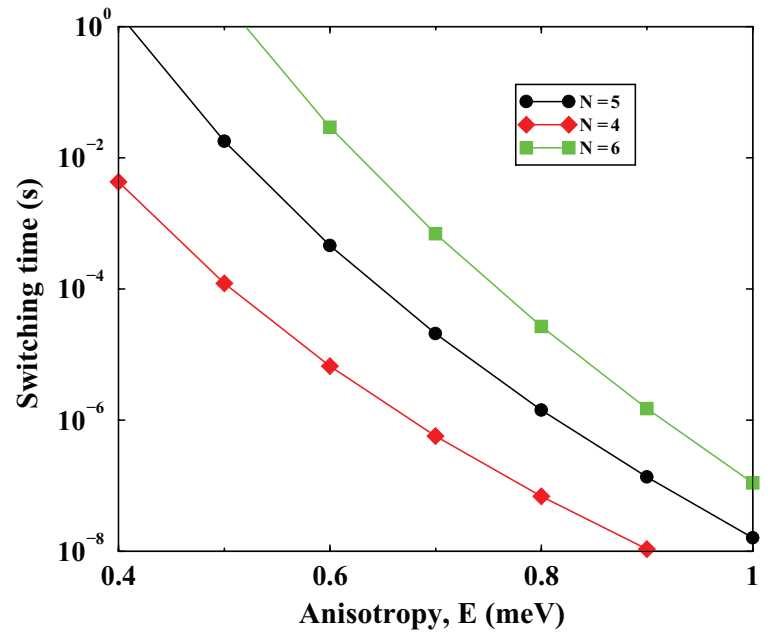

FIG. 12. (Color online) Magnetization switching time for the direct transitions induced by substrate electrons: The transition between the two extreme magnetization states occurs in a single electron collision. The magnetization switching time (inverse of the switching rate) is shown in seconds for three different chain lengths ( $N=4,5$, and 6 atoms; see insert) as a function of the transverse magnetic anisotropy of the chain, $E$. The applied B field is $1 \mathrm{~T}$.

are not very large visually (compare Figs. 9 and 11). The doubly degenerate ground state at $\mathrm{B}=0$ is split with a very tiny energy difference (around $10^{-7} \mathrm{meV}$ in Fig. 11) in the present integer spin case (it would not be in a half integer spin case ${ }^{59}$ ). For small finite $\mathrm{B}$, the two lowest states split quasilinearly with $\mathrm{B}$ and are associated with mean values of $S_{T o t, z}$ very close to \pm 10 . Actually, the anisotropy term, $E$, introduces some mixing (correlation) between the various kinds of magnetic configurations $\left|M_{1}, M_{2}, \ldots, M_{N-1}, M_{N}\right\rangle$ (where $\left|M_{i}\right\rangle$ is an eigenstate of $S_{i z}$ ), and since the two lowest states in Fig. 11 are not exactly eigenvectors of $S_{T o t, z}$, there exists a finite probability for a direct transition between the two states induced by a single collision with a substrate electron. This process is very different from the processes discussed before: The complete magnetic switching occurs in a single electron collision and not in a sequence of transitions as those discussed above; it is made possible by the correlations between magnetic configurations in the chain. However, since the mixing induced by the transverse anisotropy is not very large, the initial and final states are almost pure $\left(S_{T o t, z}= \pm 10\right)$ states, the distance between the two states in terms of number of local spin flips involved is very large, and the probability of transition induced by a single electron is very small. At low B fields, the switching time is given by the lifetime of the excited state [Eq. (6) above] and is shown in Fig. 12 as a function of the transverse anisotropy term, $E$, for an applied $\mathrm{B}$ field equal to $1 \mathrm{~T}$. The switching time increases very rapidly when $E$ decreases, and a very large anisotropy (remember $E /|D|$ is smaller than one) is required for this time to reach the nanosecond range. This switching process depends drastically on the length of the chain; indeed, the distance between the two states evaluated in terms of number of local spin flips involved in the switching increases with the number of atoms in the chain, and consequently this direct switching process slows down very rapidly when $N$ increases. For $E=0.8 \mathrm{meV}$, increasing $N$ from 5 to 6 (or decreasing $N$ from 5 to 4 ) changes the switching time by a factor around 20. This direct switch then only exists for short chains with significant transverse anisotropy. Though very slow, this process should be significant at very low temperature. At this point, one can stress that this process is different from quantum tunneling, ${ }^{4,60-62}$ which is also invoked in a magnetization switch at very low temperatures; indeed, in contrast to quantum tunneling, the present process involves (1) a collision with an electron and (2) a dissipation, i.e., an energy change between the initial and final magnetization states that is balanced by heating a substrate electron. In a classical view, there is still a barrier separating the two magnetization states, lowered by the $E$ contribution; the system does not tunnel through the barrier at constant energy, but, due to the symmetry lowering introduced by $E$, collision with a substrate electron is able to induce a direct transition between the two magnetization states. A similar process has been discussed in the context of the quantum-tunnelinginduced Kondo effect. ${ }^{59}$ It was shown that a spin-flip process (magnetic transitions induced by collision with an electron) associated with quantum tunneling of magnetization (mixing of different magnetic configurations induced by the transverse anisotropy) leads to a higher-order process able to reverse a molecular spin and thus lead to the Kondo effect. The situation is very similar to the present one: Electron collision-induced direct transitions between two states of opposite magnetization are made possible by correlation effects induced by transverse anisotropy.

Actually, this mixing between the switching states, i.e., the fact that they are not exactly the $M_{T o t}= \pm 10$ states, also perturbs the physical situation discussed here: When suddenly reversing $\mathrm{B}$, the system does not switch perfectly between the two $M_{T o t} \approx \pm 10$ states. The reversal induces the projection of one of the states on the entire spectrum, but it dominantly populates the other state. Here, we neglect this small projection effect and consider switching to occur from the $M_{T o t} \approx+10$ state.

The presence of $E$ also strongly affects the field threshold discussed in Sec. IV A, Eq. (13), above which the chain magnetization can switch via substrate electron collisions. At low field, due to the direct transitions discussed above, magnetization switch occurs; so, there is no B threshold for the magnetization switch, although the switch occurs at a very slow rate (see Fig. 12). When the applied B field increases, the initial state of the magnetization switch (init: $M_{T o t} \approx+10$ ) crosses successively the entire spectrum of magnetic states to end up as the highest one in the Zeeman limit (Fig. 11). This affects the magnetization switch in two ways. First, when the $M_{T o t} \approx+10$ state crosses another state, this introduces a new decay channel for the $M_{T o t} \approx+10$ state, and therefore it speeds up the state decay and the magnetization switch. Second, in the crossing region, possible interactions between the two states lead to an avoided crossing, and in the B range of the avoided crossing, the decay rates of the states are deeply modified. These two effects are visible in Fig. 13, which presents the lifetime of the $M_{T o t} \approx+10$ state as a function of the applied B field (anisotropy $E$ equal to $0.5 \mathrm{meV}$ in a $N=5$ chain). Note that the state lifetime is not equal to the switching time when several states are involved in the switch: 


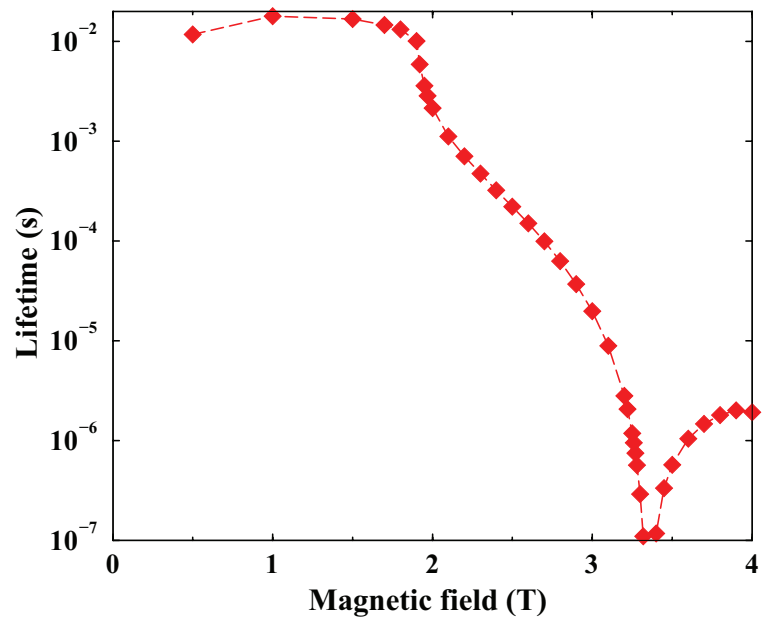

FIG. 13. (Color online) Lifetime of the upper state $\left(M_{T o t} \approx+10\right.$ state) of the magnetization switch induced by substrate electrons in a single collision event for a length chain $N=5$. The transverse magnetic anisotropy, $E$, is equal to $0.5 \mathrm{meV}$. The state lifetime (inverse of the state decay rate) is shown in seconds as a function of the applied B field in tesla.

Indeed, when several decay channels are open, the population cascades down to the ground state, but in a nonexponential way; the lifetime shown in Fig. 13 corresponds to the decay of the $M_{T o t} \approx+10$ state, irrespective of the decay final state. The lifetime of the $M_{T o t} \approx+10$ state is seen to shorten very quickly as the B field is increased, well before approaching the B threshold of the $E=0$ case. The sharp drop in the lifetime around $1.9 \mathrm{~T}$ corresponds to the first crossing of the $M_{T o t} \approx+10$ state (see Fig. 11) and to the opening of the corresponding new decay channel. The minimum in the 3.35 $\mathrm{T}$ range corresponds to a mixing between states (fifth and sixth in the increasing energy order). Beyond $4 \mathrm{~T}$, the shortening of the state lifetime goes on, but it is difficult to assign the various effects due to the very large number of state crossings involved.

The magnetization switch can also be induced by the indirect process involving higher-energy electrons, such as tunneling electrons. Figure 14 presents the corresponding probabilities (chain with $N=5$ atoms, anisotropy $D=$ $-1.5 \mathrm{meV}$ and $E=0.3 \mathrm{meV}$, applied B field: $1 \mathrm{~T}$ ) as a function of the energy of the intermediate state, referred to that of the ground state. It presents the excitation probability $P_{\text {Exc }}$ (init $\rightarrow$ $j$ ) from the initial state (init: $M_{T o t} \approx+10$ ) to all the excited states $(j)$ and the probability, $P_{\text {Ind }}($ init $\rightarrow j \rightarrow$ final $)$, for the magnetic switching induced by a tunneling electron (init $\rightarrow j$ $\rightarrow$ final), where 'final' is the ground state $\left(M_{T o t} \approx+10\right)$. The comparison with Fig. 10 illustrates the effect of the transversal anisotropy term, $E$. The number of states possibly involved in the indirect process is now very large, for any value of the B field, because $M_{T o t}$ is not a good quantum number anymore. $P_{E x c}($ init $\rightarrow j$ ) is maximum around $2 \mathrm{meV}$, corresponding to the elastic probability in the initial state. The quantized spin-wave modes that were the only excited states in Fig. 10 still dominate the excitation spectrum (init $\rightarrow j$ ), but many other states are also excited. As a result, many states

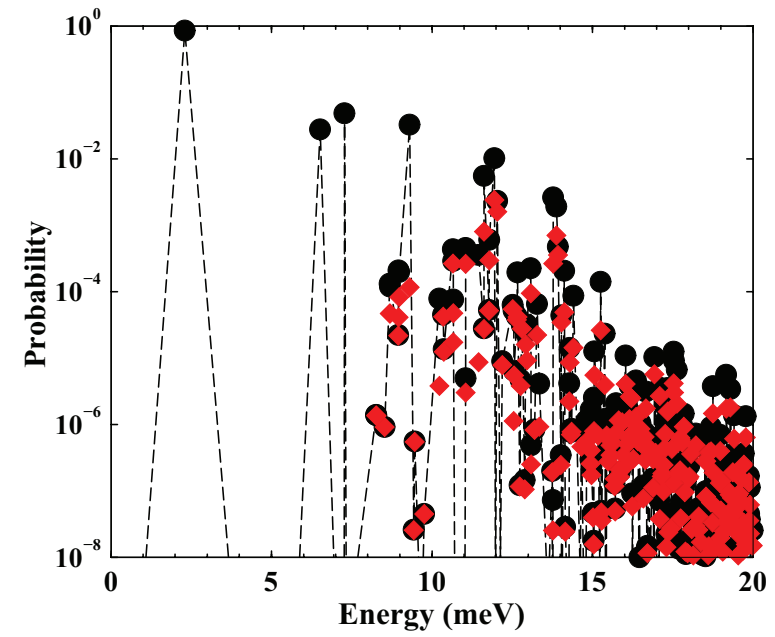

FIG. 14. (Color online) Probability for the electron-induced indirect magnetization switch process in a chain of $N=5$ atoms as a function of the intermediate state excitation energy; the anisotropy terms are $D=1.5 \mathrm{meV}$ and $E=0.3 \mathrm{meV}$, and the B field is equal to $1 \mathrm{~T}$. Black full circles joined by a dashed line: excitation probability $P_{\text {Exc }}\left(\right.$ init $\rightarrow j$ ) from the initial state (init $: M_{\text {Tot }} \approx+10$ ) to the excited state $(j)$. Red full diamonds: probability, $P_{\text {Ind }}$ (init $\rightarrow j \rightarrow$ final), for the indirect process (init $\rightarrow j \rightarrow$ final), where 'final' is the ground state $\left(M_{\text {Tot }} \approx-10\right)$. The intermediate state energies are referred to that of the ground state of the system for a vanishing field, and the largest $P_{E x c}$ (init $\rightarrow j$ ) probability around $2 \mathrm{meV}$ corresponds to the elastic probability in the initial channel.

contribute significantly to the indirect process, which does not exhibit any B threshold.

Figure 15 illustrates the variation of the indirect process probability, $P_{\text {Ind }}$ (init $\rightarrow j \rightarrow$ final), with the strength of the transverse anisotropy, $E$. For a very small $E(0.01 \mathrm{meV})$, the indirect process is basically negligible; it increases rapidly as $E$ increases: Both the indirect probability for individual states and the number of contributing intermediate states, $j$, are increasing. The two most efficient levels have an excitation energy around $12 \mathrm{meV}$, and they correspond to a mixing between a quantized spin wave and a state made of two opposite ferromagnetic domains. The variation with the transverse anisotropy term, E, is further illustrated in Fig. 16, which presents the total probability for the indirect process, given by:

$$
P_{\text {Ind }, \text { Tot }}=\sum_{j} P_{\text {Ind }}(\text { init } \rightarrow j \rightarrow \text { final }) .
$$

It gives the number of indirect transitions (init $\rightarrow$ final) per tunneling electron for an electron energy above all the inelastic thresholds. Very small at small $E$, it reaches the few $10^{-2}$ range for large $E$ values. A sharp structure can be seen in $P_{\text {Ind }, T \text { ot }}$ at small $E$. It corresponds to the crossing of two levels of the chain of different character: a quantized spin wave and a state made of two opposite ferromagnetic domains (a domain wall); these two states can be seen in Fig. 15 as the two main contributors to the indirect process. The indirect process via an intermediate level $j$ is very efficient if the $j$ state can be efficiently excited from the ground state by a tunneling electron (quantized spin-wave character associated 


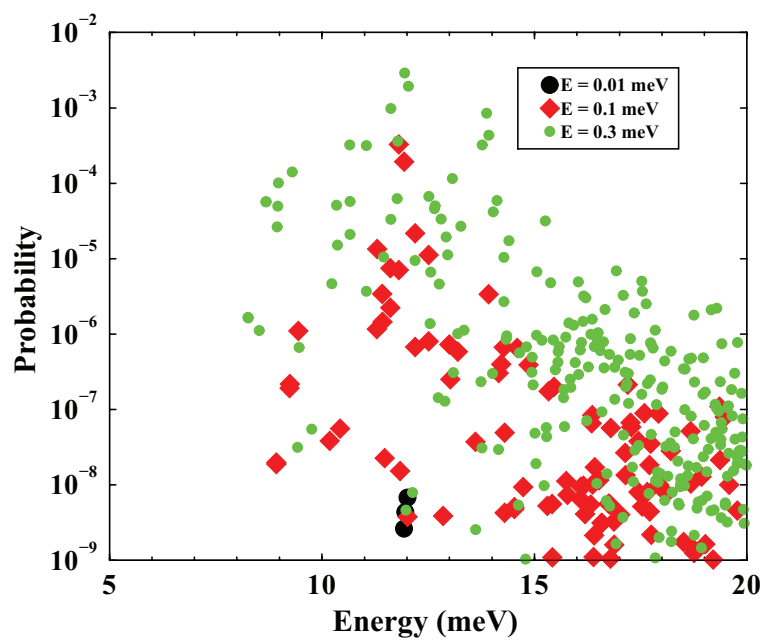

FIG. 15. (Color online) Probability for the electron-induced indirect magnetization switch process in a chain of $N=5$ atoms; the longitudinal anisotropy term is $D=1.5 \mathrm{meV}$, and the B field is equal to $1 \mathrm{~T}$. The anisotropy term, $E$, is variable (see insert). The probability, $P_{\text {Ind }}$ (init $\rightarrow j \rightarrow$ final), of the indirect process (init $\rightarrow j$ $\rightarrow$ final) is shown as a function of the energy of the intermediate state, $j$, referred to that of the ground state for a vanishing field. The magnetization switch occurs between the 'init' $\left(M_{\text {Tot }} \approx+10\right)$ state and the 'final' state $\left(M_{T o t} \approx-10\right.$; ground state).

with the initial state) and if the state $j$ decays significantly to the ground state ( $j$ state a few spin flips away from the ground state). The anisotropy term, $E$, introduces some mixing (correlation) between the various kinds of magnetic

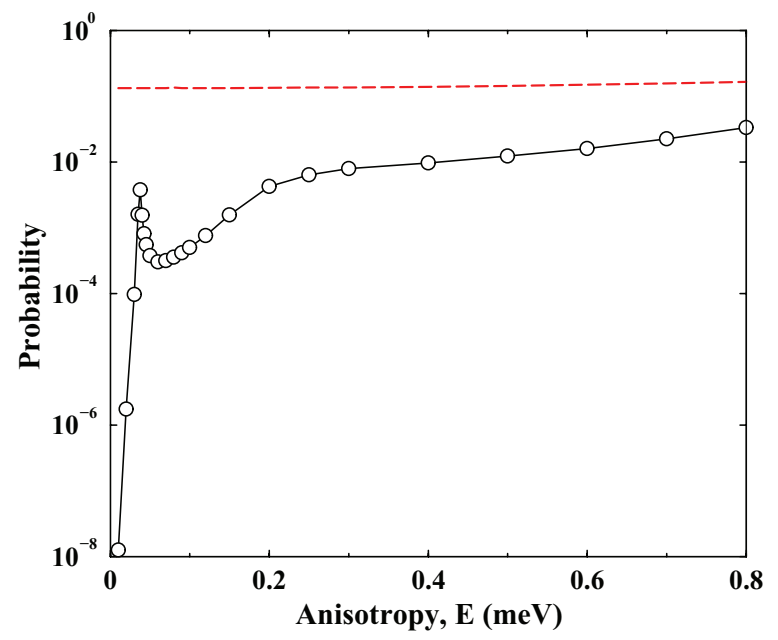

FIG. 16. (Color online) Total probability for the electron-induced indirect magnetization switch process in a chain of $N=5$ atoms; the longitudinal anisotropy term is $D=1.5 \mathrm{meV}$, and the B field is equal to $1 \mathrm{~T}$. The magnetization switch occurs between the 'init' $\left(M_{\text {Tot }} \approx\right.$ $+10)$ state and the 'final' state $\left(M_{T o t} \approx-10\right.$; ground state). The total probability, $\sum_{j} P_{\text {Ind }}$ (init $\rightarrow j \rightarrow$ final), of the indirect process for a tunneling electron with energy larger than all excitation thresholds is shown (full black line with open circles) as a function of the transverse magnetic anisotropy term, $E$. The dashed red line shows the total excitation probability $\sum_{j} P_{E x c}($ init $\rightarrow j$ ) for a tunneling electron with energy larger than all excitation thresholds. configurations $\left|M_{1}, M_{2}, \ldots, M_{N-1}, M_{N}\right\rangle$ (where $\left|M_{i}\right\rangle$ is an eigenstate of $S_{i z}$ ) and makes the indirect process possible, but still, for small anisotropies, correlation effects (mixing of configurations) are limited. However, if by accident two configurations of different characters cross for a certain $E$ value, then one can expect significant mixing and a boost of the indirect process in the corresponding $E$ range, and this occurs around $E=0.04 \mathrm{meV}$ (Fig. 15).

Figure 16 also displays the total excitation probability of the initial state by a tunneling electron: $\sum_{j} P_{E x c}$ (init $\rightarrow j$ ). First, one can see that this total excitation probability does not change much with $E$ (it slightly increases with $E$ ); the correlation simply appears to share over many levels the excitation probability that corresponds to spin waves in the limit of vanishing $E$. Half of $\sum_{j} P_{E x c}($ init $\rightarrow j$ ) can be considered as an estimate of the maximum possible probability for the indirect process: It corresponds to the case of strong correlations where an excited $j$ state decays with equal probabilities to the initial and final states. Besides the sharp structure, $P_{\text {Ind }, T o t}$ is seen to increase steadily with $E$, following the increase of correlations in the system, and indeed it approaches the above limit as $E$ increases.

A recent experiment devoted to the switching between Néel states induced by tunneling electrons in supported antiferromagnetic chains invoked the existence of both direct transitions between the Néel states and indirect processes involving spinwave excitation and domain wall creation; ${ }^{25,26}$ these processes are not simple spin-flip excitations, they are made possible by the significant correlations in the antiferromagnetic chain, in a way very similar to the switching processes discussed above. The correlation-mediated processes thus appear to exist in both ferromagnetic and antiferromagnetic coupling cases (see also a discussion in Ref. 45).

During the completion of this work, another experimentaltheoretical study appeared on tunneling electron-induced control of supported nanomagnets made of a cluster of $\mathrm{Fe}$ atoms coupled ferromagnetically. ${ }^{24}$ It invokes two types of processes described in a macrospin approximation: a spintransfer torque process in which the magnetization is stepwise changing due to electron collisions, and more direct processes involving the combined action of a spin-flip transition and of the transverse magnetic anisotropy of the macrospin. Again, this is very similar to the process discussed in the above section: A large change of magnetization can be induced in a nanomagnet in a single step, once correlation induced by the magnetic anisotropy is taken into account.

\section{CONCLUDING SUMMARY}

We have studied the quantum microscopic processes active in the magnetic reversal of a model finite-size chain of ferromagnetic atoms adsorbed on a surface when the applied magnetic field is reversed at $0 \mathrm{~K}$.

(1) The simplest case is that of a pure Heisenberg chain. At low field, the magnetic reversal implies the cascade via the sublevels of the Zeeman structure of the ground state via magnetic moment steps equal to $1 \hbar$. This corresponds to the well-known Néel-Brown description of magnetic reversal as the global rotation of a macrospin. The active process is inelastic scattering of a substrate electron leading to the creation of 
an electron-hole pair. The magnetization reversal time varies like $\log N$ ( $N$ is the number of atoms) and is independent of the details of the structure (open or closed chain, 1D or 2D structure, strength of the Heisenberg coupling).

(2) The injection of polarized electrons by an STM tip into one of the atoms of the chain speeds up the magnetic reversal. This acceleration proceeds via the excitation of spin waves in the chain, the decay of which accelerates the cascade through the Zeeman sublevels. This process is very efficient, where a very small number of injected electrons is necessary to induce the reversal, thus confirming the large torque effect of injected electrons.

(3) The presence of a negative longitudinal magnetic anisotropy modifies the magnetic reversal process. The cascade via the Zeeman sublevels is energetically impossible at low magnetic field and becomes possible above a certain threshold. Similarly, the reversal induced by injected polarized electrons requires a minimum magnetic field.

(4) The presence of a transverse magnetic anisotropy deeply modifies the magnetic reversal of the chain. The projection of the chain magnetic moment on the magnetic axis is not a good quantum number anymore, relaxing a selection rule for electron-induced transitions. As a consequence: (i) Magnetic reversal can occur under a direct transition induced by collision of a single substrate electron. This is at variance with the cascade via the Zeeman sublevels (or the Néel-Brown process), which involves many steps.

(ii) When the applied B field is increased, new decay channels appear for the upper state of the magnetization reversal that efficiently speed up the reversal.

(iii) An indirect process induced by injected electrons also exists. However, it is not limited to the spin-wave excitation, but a large number of chain states can participate in the reversal process, possibly with a number of steps smaller than in the pure Heisenberg chain case.

(iv) Both direct and indirect transitions rapidly increase with the magnitude of the magnetic anisotropy. Both transitions are mediated by the large correlations in the chain (mixing between different configurations of local spins) that make possible many electron-induced transitions between the various magnetic states in the chain.

(v) Direct and indirect transitions make possible large changes in magnetization in nanomagnets involving only a very small number of steps (even the direct magnetization reversal in a single step). These are present in both ferromagnetically and antiferromagnetically coupled nano-objects.
${ }^{1}$ L. Néel, Ann. Geophys. 5, 99 (1949).

${ }^{2}$ W. F. Brown, Phys. Rev. 130, 1677 (1963).

${ }^{3}$ W. Wernsdorfer, E. B. Orozco, K. Hasselbach, A. Benoit, B. Barbara, N. Demoncy, A. Loiseau, H. Pascard, and D. Mailly, Phys. Rev. Lett. 78, 1791 (1997).

${ }^{4}$ W. Wernsdorfer, E. B. Orozco, K. Hasselbach, A. Benoit, D. Mailly, O. Kubo, H. Nakano, and B. Barbara, Phys. Rev. Lett. 79, 4014 (1997).

${ }^{5}$ W. T. Coffey, D. S. F. Crothers, J. L. Dormann, Yu. P. Kalmykov, E. C. Kennedy, and W. Wernsdorfer, Phys. Rev. Lett. 80, 5655 (1998).

${ }^{6}$ M. Bode, O. Pietzsch, A. Kubetzka, and R. Wiesendanger, Phys. Rev. Lett. 92, 067201 (2004).

${ }^{7}$ M. Bode, A. Kubetzka, K. von Bergmann, O. Pietzsch, and R. Wiesendanger, Micro. Res. Techn. 66, 117 (2005).

${ }^{8}$ S. Krause, G. Herzog, T. Stapelfeldt, L. Berbil-Bautista, M. Bode, E. Y. Vedmedenko, and R. Wiesendanger, Phys. Rev. Lett. 103, 127202 (2009).

${ }^{9}$ S. Rohart, P. Campiglio, V. Repain, Y. Nahas, C. Chacon, Y. Girard, J. Lagoute, A. Thiaville, and S. Rousset, Phys. Rev. Lett. 104, 137202 (2010).

${ }^{10}$ S. Ouazi, S. Wedekind, G. Rodary, H. Oka, D. Sander, and J. Kirschner, Phys. Rev. Lett. 108, 107206 (2012).

${ }^{11}$ D. Hinzke and U. Nowak, Phys. Rev. B 58, 265 (1998).

${ }^{12}$ D. Hinzke and U. Nowak, Phys. Rev. B 61, 6734 (2000).

${ }^{13}$ U. Nowak, O. N. Mryasov, R. Wieser, K. Guslienko, and R. W. Chantrell, Phys. Rev. B 72, 172410 (2005).

${ }^{14}$ S. Rohart, V. Repain, A. Thiaville, and S. Rousset, Phys. Rev. B 76, 104401 (2007).

${ }^{15}$ D. A. Garanin and H. Kachkachi, Phys. Rev. B 80, 014420 (2009).

${ }^{16}$ V. L. Safonov and H. N. Bertram, Phys. Rev. B 63, 094419 (2001).

${ }^{17}$ C. Etz, M. Costa, O. Eriksson, and A. Bergman, Phys. Rev. B 86, 224401 (2012).
${ }^{18}$ M. Tsoi, A. G. M. Jansen, J. Bass, W.-C. Chiang, M. Seck, V. Tsoi, and P. Wyder, Phys. Rev. Lett. 80, 4281 (1998).

${ }^{19}$ F. J. Albert, N. C. Emley, E. B. Myers, D. C. Ralph, and R. A. Buhrman, Phys. Rev. Lett. 89, 226802 (2002).

${ }^{20} \mathrm{~S}$. Krause, L. Berbil-Bautista, G. Herzog, M. Bode, and R. Wiesendanger, Science 317, 1537 (2007).

${ }^{21}$ G. Herzog, S. Krause, and R. Wiesendanger, Appl. Phys. Lett. 96, 102505 (2010)

${ }^{22}$ S. Krause, G. Herzog, A. Schlenhoff, A. Sonntag, and R. Wiesendanger, Phys. Rev. Lett. 107, 186601 (2011).

${ }^{23}$ A. Schlenhoff, S. Krause, A. Sonntag, and R. Wiesendanger, Phys. Rev. Lett. 109, 097602 (2012).

${ }^{24}$ A. A. Khajetoorians, B. Baxevanis, C. Hübner, T. Schlenk, S. Krause, T. O. Wehling, S. Lounis, A. Lichtenstein, D. Pfannkuche, J. Wiebe, and R. Wiesendanger, Science 339, 55 (2013).

${ }^{25}$ S. Loth, S. Baumann, C. P. Lutz, D. M. Eigler, and A. J. Heinrich, Science 335, 196 (2012).

${ }^{26}$ J. P. Gauyacq, S. M. Yaro, X. Cartoixa, and N. Lorente, Phys. Rev. Lett. 110, 087201 (2013).

${ }^{27}$ A. J. Heinrich, J. A. Gupta, C. P. Lutz, and D. M. Eigler, Science 306, 466 (2004).

${ }^{28}$ C. F. Hirjibehedin, C. Y. Lin, A. F. Otte, M. Ternes, C. P. Lutz, B. A. Jones, and A. J. Heinrich, Science 317, 1199 (2007).

${ }^{29}$ N. Tsukahara, K. I. Noto, M. Ohara, S. Shiraki, N. Takagi, Y. Takata, J. Miyawaki, M. Taguchi, A. Chainani, S. Shin, and M. Kawai, Phys. Rev. Lett. 102, 167203 (2009).

${ }^{30}$ X. Chen, Y.-S. Fu, S.-H. Ji, T. Zhang, P. Cheng, X.-C. Ma, X.-L. Zou, W.-H. Duan, J.-F. Jia, and Q.-K. Xue, Phys. Rev. Lett. 101, 197208 (2008).

${ }^{31}$ J. Fransson, Nano Lett. 9, 2414 (2009).

${ }^{32}$ J. Fernandez-Rossier, Phys. Rev. Lett. 102, 256802 (2009).

${ }^{33}$ M. Persson, Phys. Rev. Lett. 103, 050801 (2009). 
${ }^{34}$ N. Lorente and J. P. Gauyacq, Phys. Rev. Lett. 103, 176601 (2009).

${ }^{35}$ S. Lounis, A. T. Costa, R. B. Muniz, and D. L. Mills, Phys. Rev. B 83, 035109 (2011).

${ }^{36}$ B. Chilian, A. A. Khajetoorians, S. Lounis, A. T. Costa, D. L. Mills, J. Wiebe, and R. Wiesendanger, Phys. Rev. B 84, 212401 (2011).

${ }^{37}$ A. Hurley, N. Baadji, and S. Sanvito, Phys. Rev. B 84, 035427 (2011).

${ }^{38}$ S. Loth, K. von Bergmann, M. Ternes, A. F. Otte, C. P. Lutz, and A. J. Heinrich, Nat. Phys. 6, 340 (2010).

${ }^{39}$ S. Loth, M. Etzkorn, C. P. Lutz, D. M. Eigler, and A. J. Heinrich, Science 329, 1628 (2010).

${ }^{40}$ H. Liu, D. Bedau, J. Z. Sun, S. Mangin, E. E. Fullerton, J. A. Katine, and A. D. Kent, Phys. Rev. B 85, 220405 (2012).

${ }^{41}$ F. D. Novaes, N. Lorente, and J. P. Gauyacq, Phys. Rev. B 82, 155401 (2010).

${ }^{42}$ A. A. Khajetoorians, S. Lounis, B. Chilian, A. T. Costa, L. Zhou, D. L. Mills, J. Wiebe, and R. Wiesendanger, Phys. Rev. Lett. 106, 037205 (2011).

${ }^{43}$ J. P. Gauyacq, N. Lorente, and F. D. Novaes, Prog. Surf. Sci. 87, 63 (2012).

${ }^{44}$ J. P. Gauyacq, F. D. Novaes, and N. Lorente, Phys. Rev. B 81, 165423 (2010).

${ }^{45}$ J. P. Gauyacq and N. Lorente, Phys. Rev. B 83, 035418 (2011).

${ }^{46}$ J. P. Gauyacq and N. Lorente, Phys. Rev. B 85, 115420 (2012).

${ }^{47}$ K. Yosida, Theory of Magnetism (Springer, Berlin, 1991).

${ }^{48}$ R. A. Abram and A. Herzenberg, Chem. Phys. Lett. 3, 187 (1969).
${ }^{49}$ D. Teillet-Billy, J. P. Gauyacq, and M. Persson, Phys. Rev. B 62, R13306 (2000).

${ }^{50}$ J. Schaffert, M. C. Cottin, A. Sonntag, H. Karacuban, C. A. Bobisch, N. Lorente, J. P. Gauyacq, and R. Möller, Nat. Mater. 12, 223 (2013).

${ }^{51}$ N. Lorente, in Dynamics, Handbook of Surface Science, edited by E. Hasselbrink and B. I. Lundquist (North Holland, Amsterdam, 2008), p. 613.

${ }^{52}$ R. B. Muniz and D. L. Mills, Phys. Rev. B 68, 224414 (2003).

${ }^{53}$ S. Lounis, A. T. Costa, R. B. Muniz, and D. L. Mills, Phys. Rev. Lett. 105, 187205 (2010).

${ }^{54}$ A. A. Khajetoorians, S. Lounis, B. Chilian, A. T. Costa, L. Zhou, D. L. Mills, J. Wiebe, and R. Wiesendanger, Phys. Rev. Lett. 106, 037205 (2011).

${ }^{55}$ A. M. Lobos, M. A. Cazalilla, and P. Chudzinski, Phys. Rev. B 86, 035455 (2012).

${ }^{56}$ F. Delgado, J. J. Palacios, and J. Fernandez-Rossier, Phys. Rev. Lett. 104, 026601 (2010).

${ }^{57}$ B. Sothmann and J. Konig, New J. Phys. 12, 083028 (2010).

${ }^{58}$ Y. Wang and L. J. Sham, Phys. Rev. B 85, 092403 (2012).

${ }^{59}$ C. Romeike, M. R. Wegewijs, W. Hofstetter, and H. Schoeller, Phys. Rev. Lett. 96, 196601 (2006).

${ }^{60}$ L. Thomas, F. Lionti, R. Ballou, D. Gatteschi, R. Sessoli, and B. Barbara, Nature 383, 145 (1996).

${ }^{61}$ M.-Carmen Miguel and E. M. Chudnovsky, Phys. Rev. B 54, 388 (1996).

${ }^{62}$ D. Gatteschi and R. Sessoli, Angew. Chem. 42, 268 (2003). 